\title{
High-Resolution Seeded Simulations of Western North Pacific Ocean Tropical Cyclones in Two Future Extreme Climates
}

\author{
J. G. MCLAY \\ Naval Research Laboratory, Monterey, California \\ E. A. HENDRICKS \\ Department of Meteorology, U.S. Naval Postgraduate School, Monterey, California \\ J. MOSKAITIS \\ Naval Research Laboratory, Monterey, California
}

(Manuscript received 31 May 2018, in final form 15 October 2018)

\begin{abstract}
A variant of downscaling is devised to explore the properties of tropical cyclones (TCs) that originate in the open ocean of the western North Pacific Ocean (WestPac) region under extreme climates. This variant applies a seeding strategy in large-scale environments simulated by phase 5 of the Coupled Model Intercomparison Project (CMIP5) climate-model integrations together with embedded integrations of Coupled Ocean-Atmosphere Mesoscale Prediction System for Tropical Cyclones (COAMPS-TC), an operational, highresolution, nonhydrostatic, convection-permitting numerical weather prediction (NWP) model. Test periods for the present day and late twenty-first century are sampled from two different integrations for the representative concentration pathway (RCP) 8.5 forcing scenario. Then seeded simulations for the present-day period are contrasted with similar seeded simulations for the future period. Reinforcing other downscaling studies, the seeding results suggest that the future environments are notably more conducive to high-intensity TC activity in the WestPac. Specifically, the future simulations yield considerably more TCs that exceed 96-kt $(1 \mathrm{kt} \approx$ $0.5144 \mathrm{~m} \mathrm{~s}^{-1}$ ) intensity, and these TCs exhibit notably greater average life cycle maximum intensity and tend to spend more time above the 96-kt intensity threshold. Also, the future simulations yield more TCs that make landfall at $>64-\mathrm{kt}$ intensity, and the average landfall intensity of these storms is appreciably greater. These findings are supported by statistical bootstrap analysis as well as by a supplemental sensitivity analysis. Accounting for COAMPS-TC intensity forecast bias using a quantile-matching approach, the seeded simulations suggest that the potential maximum western North Pacific TC intensities in the future extreme climate may be approximately $190 \mathrm{kt}$.
\end{abstract}

\section{Introduction}

Tropical cyclones (TCs) can be responsible for enormous losses in terms of both lives and property when they make landfall. For instance, three of the top four most costly natural disasters over the period 1970-2016 as measured by insured property losses were TC landfalls (namely, Hurricanes Katrina, Sandy, and Andrew; Swiss Re 2017). Hence, from the perspective of risk management, it is of great interest to understand how the physical properties and statistics of TCs might be

Corresponding author: J. G. McLay, justin.mclay@nrlmry.navy. mil different in potential future climates. This interest has spawned a steady stream of studies on the topic within the past decade. Most of the studies rely on dynamical climate forecasts from the Intergovernmental Panel on Climate Change (IPCC) phase 3 of the Coupled Model Intercomparison Project (CMIP3) and phase 5 of the Coupled Model Intercomparison Project (CMIP5; e.g., Taylor et al. 2012) and loosely fit into one of several categories. There are those that make purely statistical projections using the large-scale environments forecast by the climate models, such as Villarini and Vecchi (2013), who gauge changes in TC intensity based on statistical modeling of the power dissipation index (PDI). Second, there are studies that examine how those 
TCs that are explicitly simulated by the climate models differ between the present day and future (e.g., Yoshimura and Sugi 2005; Oouchi et al. 2006; Bengtsson et al. 2007; Knutson et al. 2008; Zhao and Held 2010; Murakami et al. 2011, 2012; Choi and Cha 2012; Camargo 2013; Roberts et al. 2015). And then there are downscaling studies that simulate TCs in future climates by embedding high-resolution numerical weather prediction (NWP) models in large-scale environments defined by the relatively low-resolution climate-model forecasts. This latter category encompasses two different approaches. In one, the climate model is relied upon to explicitly spawn TCs, and then any TCs that emerge are simulated using the embedded high-resolution model (e.g., Bender et al. 2010; Tsuboki et al. 2015; Knutson et al. 2013, 2015; Nakamura et al. 2016; Kossin et al. 2016). In the other, "seed vortices" representing nascent TCs are placed within the large-scale environments defined by the climate forecasts and then the seeds are evolved using the higherresolution model (e.g., Emanuel 2006, 2013; Emanuel et al. 2008; Hill and Lackmann 2011; Korty et al. 2017). There are some additional studies that fall outside of the above three loose categories, including one that derives trends in TC potential intensity from reanalysis data (Bister and Emanuel 2002) and one that uses an idealized model to investigate TC behavior as a function of increasing sea surface temperature (SST; Khairoutdinov and Emanuel 2013).

A number of common themes have emerged from these studies. For instance, they generally find that in the more extreme climates the strongest TCs are likely to be more intense than in present day (e.g., Tsuboki et al. 2015; Knutson et al. 2015; Emanuel et al. 2008; Emanuel 2013), and that TCs of a given intensity are likely to reach higher latitudes than in present day (e.g., Korty et al. 2017; Kossin et al. 2016). Nevertheless, the state of the science is such that there are still meaningful uncertainties in the results (e.g., Walsh et al. 2015). For instance, the results suggest that the maximum intensity of TCs is a notable function of $\mathrm{CO}_{2}$ forcing scenario, with one downscaling study based on the CMIP5 representative concentration pathway (RCP) 4.5 (RCP4.5) intermediate forcing scenario suggesting a maximum sustained surface (i.e., $10 \mathrm{~m}$ ) wind speed (MWS) of $\sim 156 \mathrm{kt}$ $\left(\sim 80 \mathrm{~m} \mathrm{~s}^{-1} ; 1 \mathrm{kt} \approx 0.5144 \mathrm{~m} \mathrm{~s}^{-1}\right)$ in the late twenty-first century (Knutson et al. 2015); another downscaling study based on the CMIP3 Special Report on Emission Scenarios (SRES) A1B intermediate forcing scenario suggesting an MWS for western North Pacific Ocean (WestPac) TCs of as much as $175 \mathrm{kt}\left(\sim 90 \mathrm{~m} \mathrm{~s}^{-1}\right.$; Tsuboki et al. 2015); and a third downscaling study based on a scenario with 8 times the $\mathrm{CO}_{2}$ of the preindustrial era suggesting an MWS of more than $210 \mathrm{kt}\left(\sim 108 \mathrm{~m} \mathrm{~s}^{-1}\right.$; Korty et al. 2017). However, there have been few studies conducted for any given forcing scenario, which leaves some question as to how characteristic the reported MWS values for the various forcing scenarios are. Many additional uncertainties arise from other factors beyond the $\mathrm{CO}_{2}$ forcing scenarios. There are large uncertainties in the climate-model forecasts themselves, with the CMIP5 project made up of 20 climate models of varying resolution and physics formulation. Additionally, there are substantial differences in the resolution and formulation of the various downscaling NWP models. For instance, the studies have variously used a model with nested primitive equation dynamics and parameterized convection (e.g., Hill and Lackmann 2011; Knutson et al. 2015; Nakamura et al. 2016); a cloud-resolving model without convective parameterization (Tsuboki et al. 2015); and an axisymmetric, hydrostatic, angular-momentum coordinate model (e.g., Emanuel 1995; Emanuel et al. 2004; Korty et al. 2017). Other uncertainties arise from differences in the ability of the relatively low-resolution climate models to explicitly represent TC genesis and even from differences in the specification of TC-tracking algorithms. The present uncertainties point to the need for continued investigation of how all aspects of TC behavior (intensity, track, duration, etc.) might change in future climates.

For its part, this study presents the results of a downscaling variant that combines a seeding methodology analogous to that of Emanuel et al. (2008) with an operational, high-resolution, nonhydrostatic, convection-permitting TC NWP model. This particular combination of seeding with a sophisticated and proven operational NWP model has not been investigated to date and thus may serve as a useful complement to the existing literature. The emphasis of this study is on TC behavior in the WestPac region, given the region's vital importance to both commercial and military maritime interests and the extensive coastal centers of infrastructure and population that are at risk from TC-landfall events. The study is organized as follows. Section 2 further describes the experimental design including the climatemodel data, the NWP model, and the seeding methodology. In section 3, results are presented with an emphasis on TC intensity and track. Section 4 presents the conclusions.

\section{Experimental design}

\section{a. Data and models}

\section{1) Climate-Model data}

The same climate-model data as in McLay et al. (2016) are employed here and the text of this section follows from that paper with minor alteration. Largescale environmental states for the future climates are sourced from the CMIP5 RCP8.5 integrations of the National Center for Atmospheric Research (NCAR) 
Community Climate System Model, version 4 (CCSM4; Gent et al. 2011), and L'Institut Pierre Simon Laplace (IPSL) Coupled Model, version 5A (CM5A; Dufresne et al. 2013). RCP8.5 simulates the extreme scenario of climate change associated with the highest estimates of anthropogenic forcing of $\mathrm{CO}_{2}$. All CCSM4 and CM5A data were downloaded from the Earth System Grid Federation (ESGF) CMIP5 data archive (Williams et al. 2011). The horizontal resolution of the CCSM4 (CM5A) data is $0.9375^{\circ} \times 1.25^{\circ}\left(2.50^{\circ} \times 1.26^{\circ}\right)$ on a latitudelongitude grid, vertical resolution is 26 (39) levels, model top is $3.54(0.04) \mathrm{hPa}$, and temporal resolution is six hours. The roughly $1^{\circ}$ horizontal resolution of the CCSM4 data is relatively high among the CMIP5 models.

CCSM4's (CM5A's) climate sensitivity is estimated to be 2.3 (3.9) $\mathrm{K}$ per $2 \times \mathrm{CO}_{2}$, which is at the low (high) end of the range for the CMIP5 models (Vial et al. 2013). The fact that the climate sensitivities of the CCSM4 and CM5A are at the opposite extremes for the CMIP5 models was a principal factor in the choice to use the CCSM4 and CM5A data. Other factors were the 6-hourly temporal resolution and the relatively high resolution of the CCSM4 data.

\section{2) TEST PERIOD SELECTION}

Two test periods are constructed, the first consisting of the months August-October for six years of climatemodel data from the present day (referred to as the "present" period) and the second consisting of AugustOctober for six years of climate-model data from the last decade of the twenty-first century (referred to as the "future" period). To give the present and future periods a multimodel complexion, each is composed of three years of CCSM4 data and three years of CM5A data. The total number of years in each period (six) is necessarily constrained by computational expense. Because of this, the selection of years for each period is guided by statistical subsampling theory and considerations of El Niño-Southern Oscillation (ENSO). ENSO is the dominant mode of interannual variability and is known to be a strong modulator of TC activity (e.g., Wang et al. 1999; Camargo et al. 2007). Regarding the subsampling theory, it is an established result (e.g., Bentley 1999) that if one has a set of $n$ values and one wants to find the subsample of size $m(m<n)$ that has the maximum possible variability of any subsample of size $m$, then one must order the $n$ values of the set from smallest to largest and select the $m / 2$ smallest values and the $m / 2$ largest values (assuming $m$ is even, for simplicity). In the present study, this subsampling theory is applied in the context of annual August-October Niño-3.4 SST anomalies with respect to an AugustOctober decadal average. These annual SST anomalies are obtained as follows using the daily SST fields from a given climate model:

1) First, the decadal average of SST for the months August-October in the Niño- 3.4 region $\left(5^{\circ} \mathrm{S}-5^{\circ} \mathrm{N}\right.$ and $170^{\circ}-120^{\circ} \mathrm{W}$ ) is computed, using daily data from the years $2006-17$ for the present period and the years 2091-2100 for the future period.

2) Second, the annual average of SST for the months August-October in the Niño-3.4 region is computed for the same years as in 1 .

3) The annual August-October SST anomaly is calculated as the difference between the annual and decadal averages.

The resulting annual SST anomalies for both the CCSM4 and CM5A models are shown in Fig. 1.

Once the August-October Niño-3.4 SST anomalies have been computed for a given model, the anomalies are ordered from most negative to most positive, in accordance with the subsampling theory. Then the year selection begins by taking a year associated with a large negative SST anomaly and a year associated with a large positive SST anomaly. These two years are then supplemented by taking a year associated with a near-zero (neutral) SST anomaly. In this way, the 3-yr subsample for a given climate model is ensured to encompass large interannual variability in the Niño-3.4 SST anomalies, including all three basic ENSO states: El Niño, La Niña, and neutral conditions. The particular years that ultimately were selected to be the basis of the main results in the study are highlighted in brackets in Fig. 1.

The annual August-October SST anomalies in the WestPac region $\left(5^{\circ}-25^{\circ} \mathrm{N}\right.$ and $\left.110^{\circ}-160^{\circ} \mathrm{E}\right)$ that accompany the Niño-3.4 SST anomalies of Fig. 1 are shown in Fig. 2. In general, the WestPac SST anomalies exhibit an inverse relationship with the Niño-3.4 anomalies, but there are exceptions. One of the more obvious is CM5A year 2010, which has a large negative Niño-3.4 anomaly (Fig. 1c) but also has a moderate negative WestPac anomaly (Fig. 2c). Considering the WestPac anomalies for the CCSM4 climate data (Figs. 2a,b), one finds that the selected present-day years $(2007,2010,2014)$ favor above-average WestPac SST anomalies, including two of the three largest positive anomaly values (Fig. 2a). Meanwhile, the selected future years $(2098,2099,2100)$ are more conservative with respect to the WestPac SST anomalies, as they include the largest negative WestPac SST anomaly (year 2099) and exclude the largest positive WestPac SST anomaly (year 2094; Fig. 2b). For the CM5A climate data (Figs. 2c,d), one finds that the selected present-day years $(2008,2010,2016)$ are fairly conservative in terms of WestPac SST anomalies, as two out of the three years have negative WestPac anomalies, 
a)

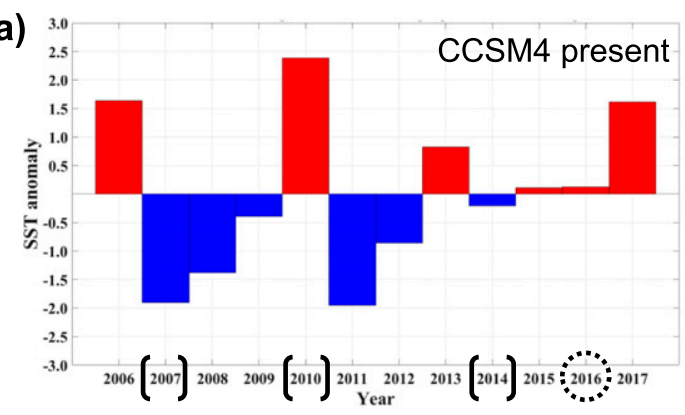

C)

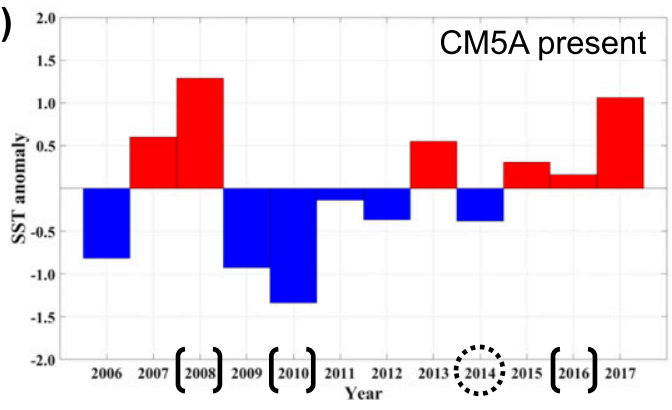

b)

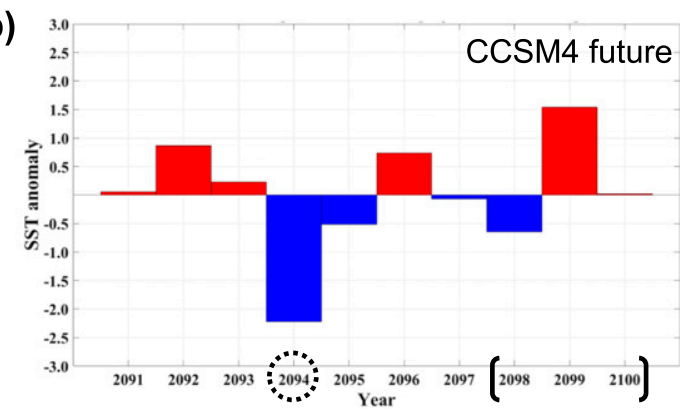

d)

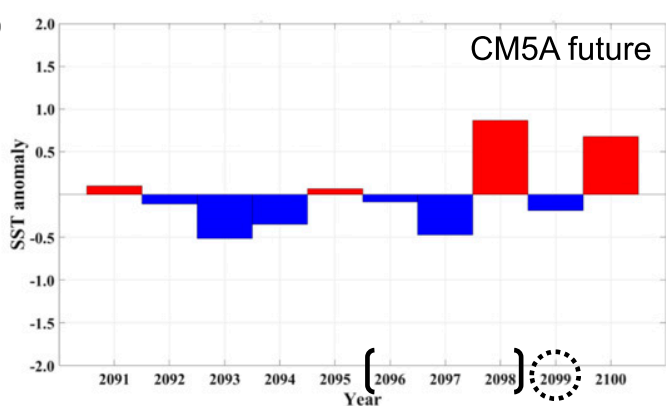

FIG. 1. (a) August-October average SST anomalies (K) with respect to the August-October decadal-mean SST for the Niño-3.4 region of the Pacific Ocean $\left(5^{\circ} \mathrm{S}-5^{\circ} \mathrm{N}\right.$ and $\left.170^{\circ}-120^{\circ} \mathrm{W}\right)$. Data are from the CCSM4 RCP8.5 climate-model integration. Decadal mean taken over the years 2006-17. Brackets indicate the years used for the main results. Dashed circles indicate the supplementary years used for the sensitivity analysis of the main results. (b) As in (a), but for the years 2091-2100. (c) As in (a), but for data from the CM5A RCP8.5 climate-model integration. (d) As in (c), but for the years 2091-2100.

including the largest negative anomaly (year 2008; Fig. 2c). The year with the largest positive anomaly is excluded (year 2014). The CM5A future years are similarly conservative: Two of the three are associated with

a)

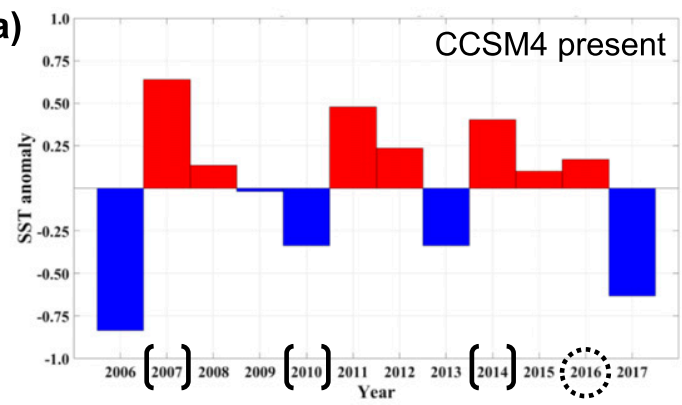

c)

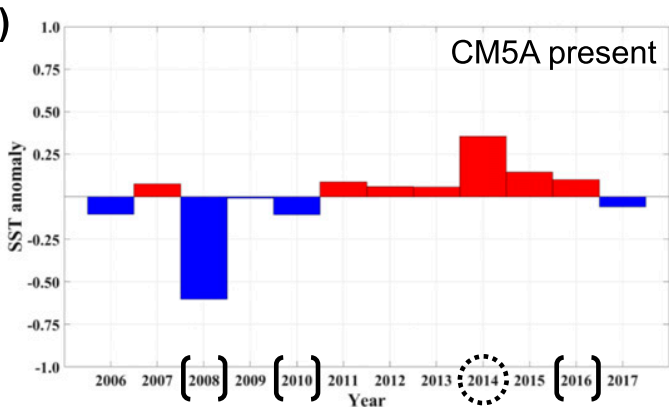

negative WestPac anomalies, and the year with the largest positive anomaly (2099) is excluded (Fig. 2d). Since the selection for the CCSM4 future years and both the CM5A present and future years happen to be fairly
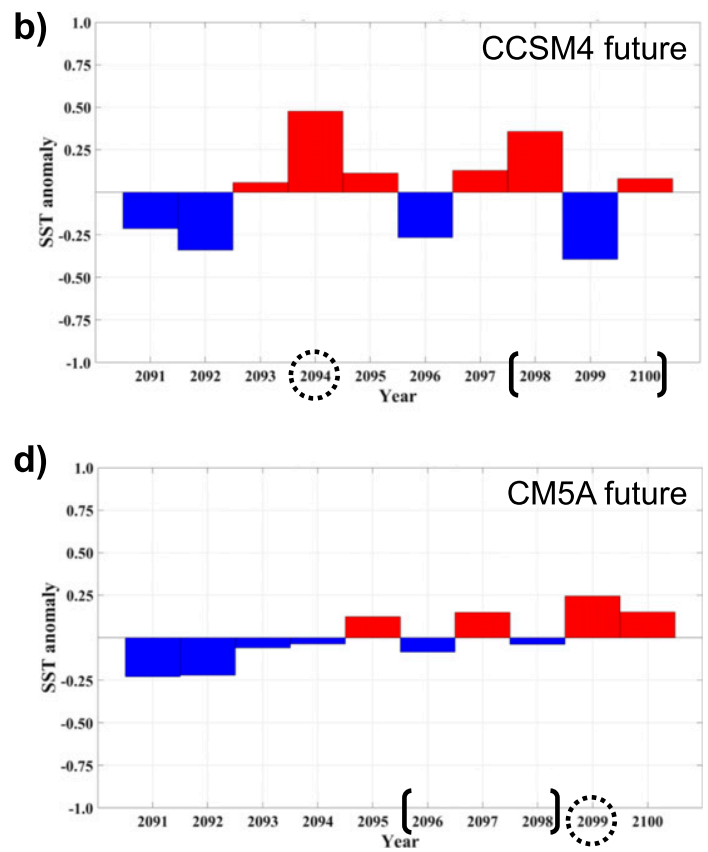

FIG. 2. As in Fig. 1, but for the WestPac region of the Pacific Ocean $\left(5^{\circ} \mathrm{N}-25^{\circ} \mathrm{N}\right.$ and $\left.110^{\circ}-160^{\circ} \mathrm{E}\right)$. 
conservative in terms of WestPac SST anomalies, an additional four years are selected to facilitate a sensitivity analysis of the main results. These supplemental years encompass the largest positive WestPac SST anomaly within the CCSM4 2091-2100 data (year 2094), the largest within the CM5A 2091-2100 data (year 2099), and the largest within the CM5A 2006-17 data (year 2014). The set of supplemental years is rounded out by CCSM4 year 2016, since the CCSM4 present years already include multiple large positive WestPac SST anomalies.

\section{3) COAMPS-TC MESOSCALE NWP MODEL}

NWP integrations of the TCs are carried out using the Coupled Ocean-Atmosphere Mesoscale Prediction System for Tropical Cyclones (COAMPS-TC; Doyle et al. 2014). COAMPS-TC is a gridded, primitive equation, nonhydrostatic, convection-permitting model. As the operational TC model for the U.S. Navy, it is run in real time in every ocean basin worldwide and is one of the leading operational tropical cyclone prediction models. The model configuration for this application is similar to the 2013 operational version of the model and includes the following features: (i) initialization based on assimilation of synthetic observations of a balanced TC vortex defined by the operational warning message, (ii) TC-following nested inner grids using an automatic TC tracker, (iii) dissipative heating (Jin et al. 2007), TC planetary boundary layer formulation using a 1.5 -order turbulence kinetic energy closure, and (iv) a surface drag parameterization for the high wind speed regime (modified from Donelan et al. 2004). A moving-nest grid configuration is used, with horizontal resolutions of 45,15 , and $5 \mathrm{~km}$ for the outer fixed grid, intermediate moving-nest grid, and inner moving-nest grid, respectively. The outer fixed grid is of dimension $12996 \mathrm{~km} \times$ $6876 \mathrm{~km}$ and spans roughly the longitudes $70^{\circ} \mathrm{E}-160^{\circ} \mathrm{W}$ and the latitudes $5^{\circ} \mathrm{S}-52^{\circ} \mathrm{N}$. The intermediate and inner moving-nest grids are 1800 and $900 \mathrm{~km}$ on a side, respectively. The forecast system also has a capability for perturbed physics parameters, but for this study, the model is run with fixed parameters. Each COAMPS$\mathrm{TC}$ integration is run for $360 \mathrm{~h}$ in order to capture the full life cycle of any resulting TC. Boundary conditions for the COAMPS-TC integrations are provided by the climate-model integrations described above, interpolated to $1.0^{\circ} \times 1.0^{\circ}$ horizontal resolution and updated at 6-hourly intervals.

\section{4) PARAmeterized ATMOSPHERE-OCEAN COUPLING}

To better represent atmosphere-ocean interactions, COAMPS-TC simulations can be run either fully coupled to the Navy Coastal Ocean Model (NCOM) or standalone with a parameterized atmosphere-ocean coupling. The parameterized coupling represents the effect of atmosphere-induced ocean mixing by cooling the SST at a rate that is linearly proportional to the wind speed at those locations where the wind speed exceeds $20 \mathrm{~m} \mathrm{~s}^{-1}$. Though simple, the parameterized coupling produces TC cold wakes with realistic gross spatial structure including, for instance, asymmetry around the TC center in cases where the TC wind field is asymmetric. Further, simulations using the parameterized coupling have proven surprisingly competitive with fully coupled simulations in comprehensive homogeneous comparisons of TC track and intensity, as Figs. 3 and 4 show. These figures denote the COAMPS-TC simulation with full coupling (CTCX) and the COAMPS-TC simulation with parameterized coupling (CTUX). The CTCX and CTUX simulations were each run for all TCs observed in the western Pacific, eastern Pacific, and Atlantic Ocean basins in the period 2016/17. Other than the coupling, the configuration of CTCX and CTUX are the same and mimic the operational version of COAMPS-TC for the years in question. For instance, the horizontal resolution of the innermost nested grid for the 2016 (2017) simulations is 5 (4) km. Considering first the western Pacific Ocean basin (Fig. 3), one finds that CTUX is comparable or slightly better than CTCX in terms of track and intensity mean absolute error (MAE; Figs. 3a,b, respectively). Also, the intensity mean error (ME) of CTUX is of comparable magnitude to that of CTCX, but CTUX tends to overpredict the intensity of TCs by $3-5 \mathrm{kt}$, while CTCX tends to underpredict the intensity by a similar amount (Fig. 3b). The collective results for the western Pacific, eastern Pacific, and Atlantic Ocean basins are much the same as those for the western Pacific (Fig. 4). Notably, CTUX has smaller intensity mean error than CTCX at most lead times (Fig. 4b). Thus, in general, CTUX is a capable proxy for the fully coupled system. The parameterized coupling is also far less expensive than the full coupling and, on account of various technical obstacles, far easier to deploy with the climate-model data than the full coupling. For these reasons, this study employs the parameterized coupling to represent atmosphere-ocean interactions.

It is emphasized that the parameterized coupling does not contain any model of ocean structure. Thus, the parameterized coupling is not expected to perform as well as a fully coupled system in situations where a TC moves relatively slowly over a shallow ocean mixed layer. Such situations encompass a small minority of cases for the present day. However, recent literature suggests there is some possibility that the future climate will exhibit enhanced ocean stratification with an attendant stronger ocean coupling effect (e.g., Huang 

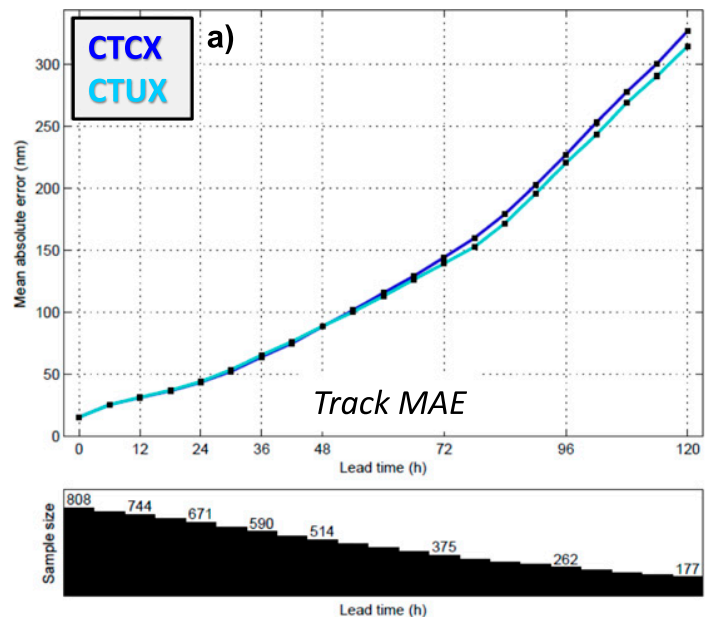

FIG. 3. (a) Homogeneous comparison of track MAE for real-world TC cases in the WestPac basin during 2016/17

simulated by CTCX and CTUX. See text for further details. (b) As in (a), but for intensity MAE and ME. et al. 2015; Tuleya et al. 2016). Since the parameterized coupling has no awareness of the ocean vertical structure, it may underestimate the reduction in intensification arising from the ocean coupling effect for those cases in the future period. This point is discussed further in the conclusions.

\section{b. Methodology}

\section{1) TC SEEDING}

The seeding approach of this study is conceptually similar to that of Emanuel et al. (2008), Emanuel (2013), and Korty et al. (2017), but is technically different in certain respects. Namely, the TC seeds are not randomly distributed in time and space, and each seed is introduced into the large-scale environment using variational data assimilation. Regarding the temporal nature
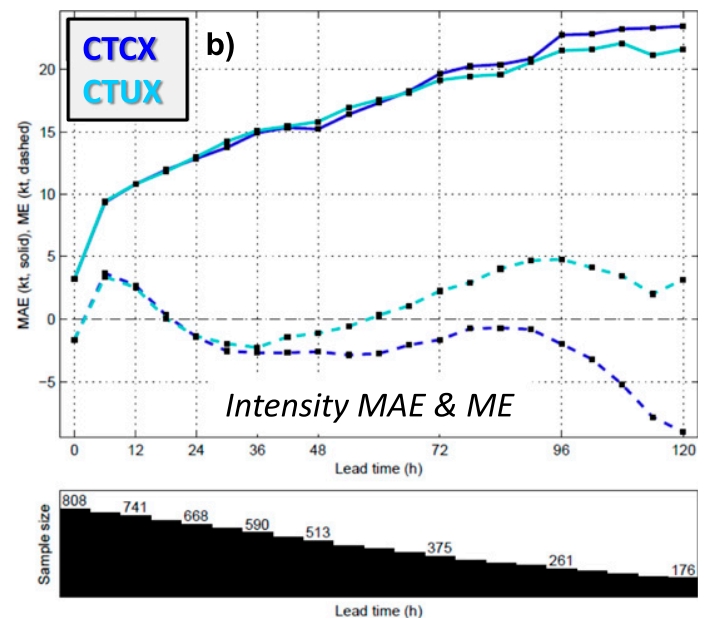

of the seeding, for a given year 15 seeds are introduced every five days over the period 1 August-31 October. This results in a total of 285 separate TC simulations for a given year and accounts for intraseasonal variability in the large-scale environmental conditions. Regarding the location of the seeding, the seeds are restricted to a subjectively chosen latitude-longitude box in the WestPac defined by the latitude band $9^{\circ}-17^{\circ} \mathrm{N}$ and the longitude band $135^{\circ}-175^{\circ} \mathrm{E}$. Within this box, seeds are introduced at fixed $10^{\circ}$ intervals in longitude and $4^{\circ}$ intervals in latitude, as illustrated in Fig. 5a. Each seed takes the form of a synthetic vortex of either 25- or 35-kt strength, depending on the simulation configuration [see section $2 b(3)$, below]. This synthetic vortex is combined with the large-scale environment using a three-dimensional variational data assimilation (3DVAR) approach, as described in the next section. It should
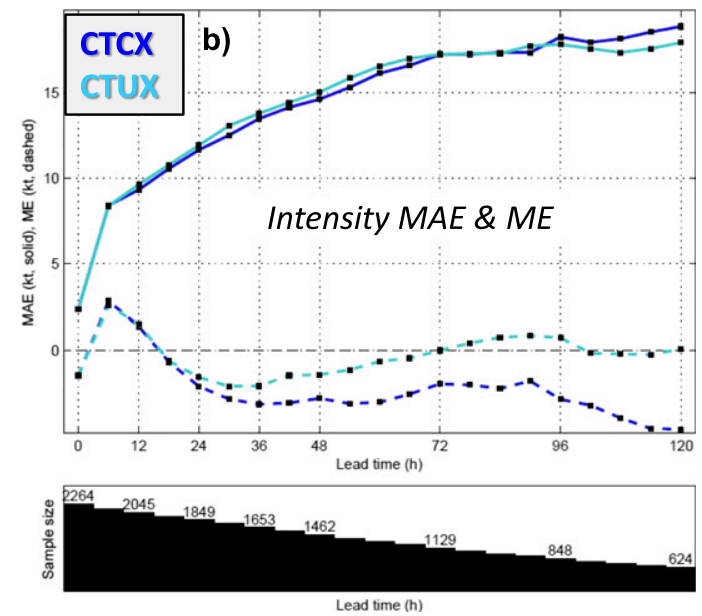

FIG. 4. As in Fig. 3, but for real-world TC cases in the Atlantic, eastern Pacific, and WestPac basins during 2016/17. 
a)

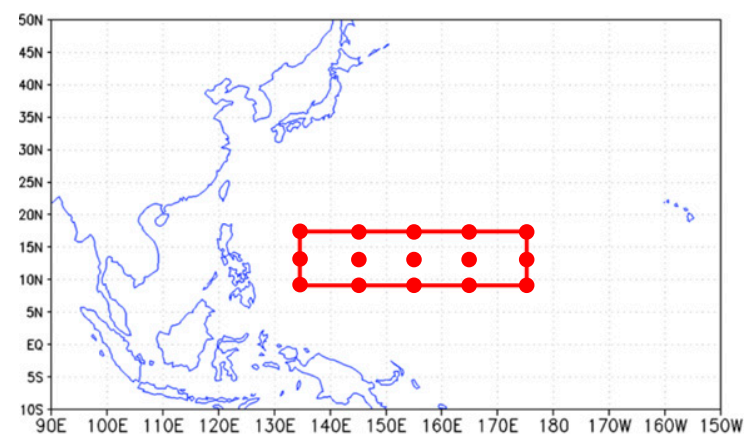

b)

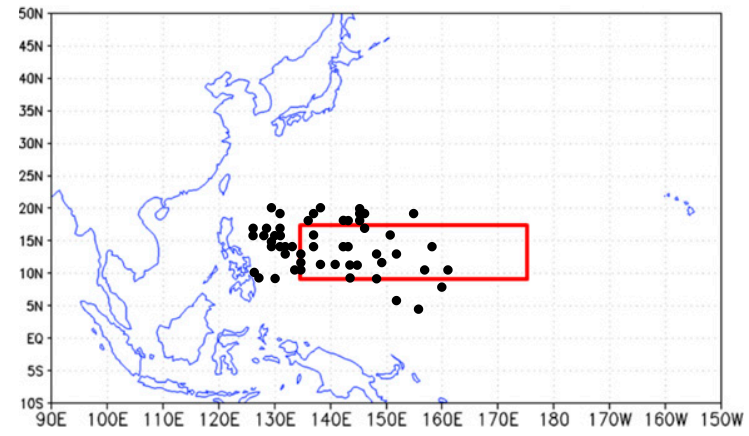

FIG. 5. (a) Latitude-longitude bounds of the WestPac seeding region (red box) and the fixed seeding locations (red-filled circles). (b) Initialization locations (black-filled circles) of the real cases for 2008-14 in relation to the seeding region.

be emphasized that no attempt was made to optimize the choice of the WestPac seeding region with respect to the genesis index or the potential intensity metric. In this respect, the results of the seeding may be conservative. Further, to be absolutely clear, each COAMPS-TC seeded forecast integration contains only one seed vortex within its initial conditions. Since there is only one seed vortex per integration, it is impossible to have interaction among seed vortices. It follows that for any given date in the test period, 15 different COAMPS$\mathrm{TC}$ integrations are performed, one for each of the 15 seeds for the date.

A question that arises with respect to the seeding approach is how often and to what effect the seeded vortices may interact with tropical vortices that are native to the climate-model data. To address this question, the time series of daily climate-model analyses were examined for the presence of native tropical vortices. It was found that the climate-model integrations employed for this study only have a very sparing number of what can be considered native TCs in the WestPac seeding region, likely because of the relatively low resolution of the climate models. For example, for year 2098 of the CCSM4 data, there are only three distinct instances in the entire period of August-October where a native vortex in or near the seeding region is associated with a mean sea level pressure (MSLP) value less than $1000 \mathrm{hPa}$ or an $850-\mathrm{hPa}$ absolute vorticity value greater than $1 \times 10^{-4} \mathrm{~s}^{-1}$. Likewise, for year 2097 of the CM5A data, there are zero instances where a native vortex in or near the seeding region is associated with an MSLP value less than $1004 \mathrm{hPa}$, and there are only four distinct instances where a native vortex in or near the seeding region is associated with an $850-\mathrm{hPa}$ absolute vorticity value greater than $1 \times$ $10^{-4} \mathrm{~s}^{-1}$. These findings indicate that on any given seeding date, it is very unlikely that a noteworthy native vortex is present in or near the seeding region.

In those rare instances when a native vortex does happen to impinge on the seeding region, the variational initialization of the seed vortex (described in the next section) will effectively eliminate the native vortex if the native vortex is within roughly $6^{\circ}$ latitude or longitude of the seed. Otherwise, if the native vortex is farther away from the seed then, just as in the real world, there can be a Fujiwhara interaction (Fujiwhara 1921) between the seed vortex and the native vortex.

\section{2) TC VARIATIONAL INITIALIZATION}

The TC structure and intensity for a given seed is initialized using synthetic observations and variational data assimilation, as described in Liou and Sashegyi (2012) and Hendricks et al. (2013). The following is a brief overview of the method. The synthetic observations are first constructed from a balanced modified Rankine vortex that is fit to the radius of maximum winds, radius of 34-kt winds, and the maximum sustained winds reported by the TC warning center. The synthetic observations of zonal and meridional velocity, geopotential height, and temperature are created at eight azimuthal segments and at radii of $0.5^{\circ}, 1^{\circ}, 2^{\circ}, 4^{\circ}$, and $6^{\circ}$ from the vortex center. These synthetic observations are extended upward from 1000 to $400 \mathrm{hPa}$ with a prescribed vertical decay that is typical of a TC-like vortex. The environmental flow is then added to the synthetic observations, and these observations are given small errors so that they are weighted more strongly in the data assimilation. Finally, the 3DVAR system is used to optimally blend the TC synthetic observations and all other conventional and satellite observations to obtain the initial fields of the model. Further details on the initialization procedure can be found in Liou and Sashegyi (2012). Note that this initialization scheme was used in the real-time version of COAMPS-TC until 2014.

\section{3) Conservative And Aggressive SIMULATION CONFIGURATIONS}

The seeding experiments are repeated in their entirety for two different simulation configurations: In the 
first, referred to as the "conservative" configuration, the seeding is carried out with $25-\mathrm{kt}$ seed vortices and the ocean mixing parameterization is turned on during the COAMPS-TC integrations. In the second, referred to as the "aggressive" configuration, the seeding is carried out with 35-kt seed vortices and the ocean mixing parameterization is turned off during the COAMPSTC integrations. Everything else is the same between the two configurations. The $25-\mathrm{kt}$ seed vortices of the conservative configuration are the same strength as those employed by Emanuel et al. (2008) and Korty et al. (2017). As compared to a 35-kt seed, a 25-kt seed should be less inclined to intensify, since among other things its weaker winds will be less supportive of airsea fluxes and its vortex will be less resilient to environmental perturbations (given its smaller reservoir of angular momentum and other quantities). For its part, the ocean mixing parameterization has a pronounced dampening effect on intensification. This is illustrated by Fig. 6, which shows the distribution of life cycle maximum intensity for two different seededsimulation sets based on environments from the CM5A data. Each simulation set comprises a total of 840 seeded simulations, generated from $25-\mathrm{kt}$ seed vortices using the methodology described above. However, for one set, the COAMPS-TC integrations are conducted with the ocean mixing parameterization turned on, and for the other, the ocean mixing parameterization is turned off. Considering either the results for the present years (Fig. 6a) or the future years (Fig. 6b), one finds that the activation of the ocean mixing parameterization leads to a notable 6-7 kt reduction in the average maximum intensity. Further, the ocean mixing parameterization notably reduces the size of the upper tail of the intensity distribution, such that the maximum intensity of the distribution is reduced by $10 \mathrm{kt}$ or more.

\section{4) Simulation Of REAL-WORLd CASES}

As an additional point of reference for the seeded TC simulations is a set of simulations of WestPac TCs that were observed over the period 2008-14. These realworld cases are simulated using COAMPS-TC in the same model configuration (resolution, nested-grid definitions, etc.) as for the seeded cases but with analyzed initial and boundary conditions from the Navy Global Environmental Model (NAVGEM; Hogan et al. 2014). The NAVGEM fields take the form of $1^{\circ} \times 1^{\circ}$ latitude-longitude grids that are updated every $6 \mathrm{~h}$. To qualify for simulation, a given observed TC must meet the criteria that (i) its life cycle occurs in the months July-November and (ii) it first reaches tropicalstorm intensity ( $>35-\mathrm{kt}$ surface wind speed) in the near
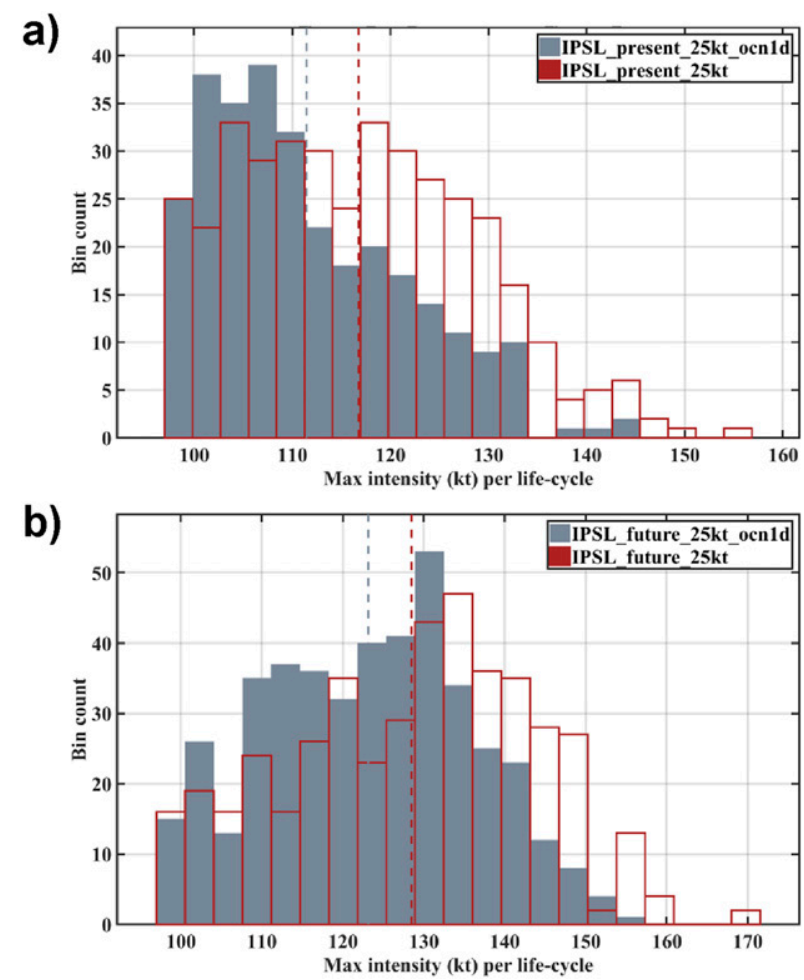

FIG. 6. (a) Distribution of life cycle maximum intensity (kt) for seeded simulations in the present years using the CM5A data, 25-kt seed vortices, and the ocean mixing parameterization turned on (gray) and turned off (red). (b) As in (a), but for seeded simulations in the future years.

vicinity of the WestPac seeding box described above in section $2 b(1)$. These criteria ensure that both the seeded storms and the real-world storms share the same time of year and area of origin, but they are also fairly restrictive. Over the 2008-14 July-November period, there are 55 observed storms that meet these criteria, or roughly $50 \%$ of the total of 115 observed storms that reach tropical-storm intensity in the WestPac during the period. These storms are detailed in Table 1. The initialized position and wind radii of each real-world TC are the same as the observed TC position and wind radii. The initialized positions of the real-world TCs are shown in Fig. 5b. Also of importance, the initialization time of each present-day TC simulation is taken to be the time at which the observed TC (according to the best track data; Knapp et al. 2010) first reaches at least $35 \mathrm{kt}$. Furthermore, the real-world TC simulations are run with the ocean mixing parameterization turned off. Since the initialized intensity of the real-world TCs is $35 \mathrm{kt}$ and the ocean mixing is turned off, the real-world TC simulations should be considered counterparts to the seeded simulations run with the aggressive configuration. 
TABLE 1. Background information of the real-world cases that were simulated for the study.

\begin{tabular}{|c|c|c|c|c|c|}
\hline \multirow[b]{2}{*}{ Year } & \multirow[b]{2}{*}{$\mathrm{TC}$} & \multirow[b]{2}{*}{ Name } & \multicolumn{3}{|c|}{ First time reaching at least $35 \mathrm{kt}$} \\
\hline & & & Time (UTC) & Lat $\left({ }^{\circ} \mathrm{N}\right)$ & Lon $\left({ }^{\circ} \mathrm{E}\right)$ \\
\hline 2008 & $13 \mathrm{~W}$ & Nuri & 120017 Aug 2008 & 16.1 & 136.7 \\
\hline 2008 & $15 \mathrm{~W}$ & Sinlaku & 12008 Sep 2008 & 16.3 & 125.8 \\
\hline 2008 & $18 \mathrm{~W}$ & Hagupit & 060019 Sep 2008 & 14.1 & 134.2 \\
\hline 2008 & $19 \mathrm{~W}$ & Jangmi & 000024 Sep 2008 & 11.7 & 138.0 \\
\hline 2008 & $21 \mathrm{~W}$ & Higos & 120029 Sep 2008 & 9.1 & 129.5 \\
\hline 2009 & $11 \mathrm{~W}$ & Vamco & 120017 Sep 2009 & 14.2 & 158.1 \\
\hline 2009 & $13 \mathrm{~W}$ & Dujuan & 12004 Sep 2009 & 19.0 & 130.9 \\
\hline 2009 & $15 \mathrm{~W}$ & Choi-wan & 180012 Sep 2009 & 15.6 & 150.7 \\
\hline 2009 & $19 \mathrm{~W}$ & Parma & 120028 Sep 2009 & 9.3 & 142.5 \\
\hline 2009 & $20 \mathrm{~W}$ & Melor & 180029 Sep 2009 & 11.0 & 157.0 \\
\hline 2009 & $22 W$ & Lupit & 000015 Oct 2009 & 11.7 & 144.4 \\
\hline 2009 & $23 \mathrm{~W}$ & Mirinae & 120026 Oct 2009 & 13.2 & 148.3 \\
\hline 2009 & $26 \mathrm{~W}$ & Nida & 060023 Nov 2009 & 8.5 & 147.8 \\
\hline 2010 & $03 \mathrm{~W}$ & Conson & 180011 Jul 2010 & 14.1 & 131.6 \\
\hline 2010 & $08 \mathrm{~W}$ & Kompasu & 180028 Aug 2010 & 19.5 & 137.7 \\
\hline 2010 & $12 \mathrm{~W}$ & Fanapi & 000015 Sep 2010 & 20.0 & 128.5 \\
\hline 2010 & $13 \mathrm{~W}$ & Malakas & 000021 Sep 2010 & 18.1 & 145.1 \\
\hline 2010 & $15 \mathrm{~W}$ & Megi & 060013 Oct 2010 & 11.8 & 141.2 \\
\hline 2010 & $16 \mathrm{~W}$ & Chaba & 180024 Oct 2010 & 16.4 & 131.1 \\
\hline 2011 & 08W & Ma-on & 060012 Jul 2011 & 18.7 & 155.2 \\
\hline 2011 & $11 \mathrm{~W}$ & Muifa & 060028 July 2011 & 11.9 & 135.4 \\
\hline 2011 & $14 \mathrm{~W}$ & Nanmadol & 120023 Aug 2011 & 15.7 & 127.5 \\
\hline 2011 & $20 \mathrm{~W}$ & Nesat & 180023 Sep 2011 & 13.9 & 137.3 \\
\hline 2011 & $22 \mathrm{~W}$ & Nalgae & 180027 Sep 2011 & 19.0 & 136.9 \\
\hline 2011 & $23 \mathrm{~W}$ & Banyan & 120011 Oct 2011 & 8.9 & 126.9 \\
\hline 2012 & $10 \mathrm{~W}$ & Saola & 180028 Jul 2012 & 17.0 & 126.1 \\
\hline 2012 & $14 \mathrm{~W}$ & Kai-tak & 060013 Aug 2012 & 16.6 & 128.1 \\
\hline 2012 & $16 \mathrm{~W}$ & Bolaven & 060020 Aug 2012 & 17.6 & 141.7 \\
\hline 2012 & $17 \mathrm{~W}$ & Sanba & 060011 Sep 2012 & 11.2 & 133.9 \\
\hline 2012 & $18 \mathrm{~W}$ & Jelawat & 180020 Sep 2012 & 13.4 & 131.6 \\
\hline 2012 & $20 \mathrm{~W}$ & Maliksi & 12001 Oct 2012 & 18.7 & 145.4 \\
\hline 2012 & $22 \mathrm{~W}$ & Prapiroon & 18007 Oct 2012 & 17.9 & 136.1 \\
\hline 2012 & $23 \mathrm{~W}$ & Maria & 120014 Oct 2012 & 17.9 & 142.9 \\
\hline 2012 & $24 \mathrm{~W}$ & Son-Tinh & 000024 Oct 2012 & 9.8 & 126.3 \\
\hline 2012 & $26 \mathrm{~W}$ & Bopha & 000027 Nov 2012 & 4.2 & 155.9 \\
\hline 2013 & $07 \mathrm{~W}$ & Soulik & 00008 Jul 2013 & 19.2 & 145.5 \\
\hline 2013 & $11 \mathrm{~W}$ & Utor & 12009 Aug 2013 & 13.6 & 132.8 \\
\hline 2013 & $17 \mathrm{~W}$ & Usagi & 120017 Sep 2013 & 17.4 & 131.0 \\
\hline 2013 & $19 \mathrm{~W}$ & Pa buk & 060021 Sep 2013 & 20.0 & 145.0 \\
\hline 2013 & $22 \mathrm{~W}$ & Fitow & 180030 Sep 2013 & 14.1 & 132.1 \\
\hline 2013 & $23 \mathrm{~W}$ & Danas & 06004 Oct 2013 & 16.8 & 145.8 \\
\hline 2013 & $24 \mathrm{~W}$ & Nari & 12009 Oct 2013 & 14.5 & 129.0 \\
\hline 2013 & $25 \mathrm{~W}$ & Wipha & 180010 Oct 2013 & 13.5 & 143.0 \\
\hline 2013 & $26 \mathrm{~W}$ & Francisco & 120016 Oct 2013 & 11.6 & 143.4 \\
\hline 2013 & $28 \mathrm{~W}$ & Krosa & 180020 Oct 2013 & 10.9 & 160.8 \\
\hline 2013 & $29 \mathrm{~W}$ & Lekima & 000030 Oct 2013 & 16.4 & 129.8 \\
\hline 2013 & $31 \mathrm{~W}$ & Haiyan & 00004 Nov 2013 & 6.1 & 151.5 \\
\hline 2014 & $08 \mathrm{~W}$ & Neoguri & 18003 Jul 2014 & 11.6 & 143.1 \\
\hline 2014 & 09W & Rammasun & 120012 Jul 2014 & 13.7 & 141.9 \\
\hline 2014 & $10 \mathrm{~W}$ & Matmo & 180017 Jul 2014 & 10.5 & 135.2 \\
\hline 2014 & $11 \mathrm{~W}$ & Halong & 180028 Jul 2014 & 12.0 & 148.7 \\
\hline 2014 & $15 \mathrm{~W}$ & Kalmaegi & 060012 Sep 2014 & 13.7 & 131.2 \\
\hline 2014 & $18 \mathrm{~W}$ & Phanfone & 000029 Sep 2014 & 12.7 & 152.1 \\
\hline 2014 & $19 \mathrm{~W}$ & Vongfong & 00003 Oct 2014 & 8.0 & 159.5 \\
\hline 2014 & $20 \mathrm{~W}$ & Nuri & 120031 Oct 2014 & 12.8 & 135.3 \\
\hline
\end{tabular}

\section{5) QUANTILE MAPPING OF INTENSITY}

The version of COAMPS-TC used for this study dates to 2013 and is known to have an intensity bias, namely, it tends to underforecast the intensity of the strongest TCs. Such an intensity bias is not unusual for NWP models of the 2013 time frame. Here in section 3h, a simple method called quantile mapping is used to account for this intensity bias. In quantile mapping, the historical COAMPS-TC intensity forecasts are partitioned into equally populated bins whose edges are the quantiles of the distribution of intensity forecasts. The verifying (i.e., best track) TC intensities corresponding to the forecast intensities are then partitioned in the same fashion. Then for each bin, the bin average of forecast intensity and the bin average of verifying intensity are calculated. One can then fit a function to the collection of binaverage values as a means to quantify the relationship between the quantiles of the forecast and verifying intensity distributions. While simple, quantile mapping has proven to be a very effective postprocessing tool for raw TC intensity forecasts (e.g., Alessandrini et al. 2018). Note that the quantile mapping application is limited strictly to the discussion in section $3 \mathrm{i}$ and that the forecast intensities presented elsewhere in the paper are raw (uncorrected) forecast intensities.

\section{6) NONPARAMETRIC TEST OF STATISTICAL SIGNIFICANCE}

To provide some statistical perspective, a test of significance is applied to most results. A consideration in the design of the test is that dependence is to be expected among the seeded cases. Specifically, there is dependence among the 15 seeded storms for any given seeding date, and there likely is dependence among the storms on successive seeding dates since these dates are separated in time by only five days. A subsampling approach is taken with respect to the seeded cases to mitigate the influence of this dependence on the significance test. To elaborate, if $n_{\text {present }}\left(n_{\text {future }}\right)$ is the total number of available seeded cases for the six present years (future years) and $n$ is the size of any given random sample from the available seeded cases, then the subsampling approach by design employs the constraint $n \ll$ $n_{\text {present(future) }}$. The relatively small size of the subsample [i.e., $n \ll n_{\text {present(future) }}$ ] makes it likely that the subsample will exhibit considerably less dependence than the full set of $n_{\text {present }}\left(n_{\text {future }}\right)$ seeded cases. For this study, using the conservative (aggressive) seeding configuration, the value of $n_{\text {present }}$ for those seeded cases that satisfy the criterion that the life cycle maximum intensity exceeds $96 \mathrm{kt}$ is 635 (1122). The corresponding value of $n_{\text {future }}$ is 795 (1245). Given these values of $n_{\text {present(future) }} n$ is 
conservatively chosen to be $n=100$, or at most $16 \%$ of the total number of available seeded cases.

Given the choice of $n=100$, the procedure of the nonparametric significance test is as follows. The description uses the "life cycle maximum intensity" metric for illustration, but the procedure easily generalizes to other metrics such as "intensity at first landfall" and "storm speed."

1) A large number $M$ of random trials are performed where for each trial $i(i \in\{1, \ldots, M\})$ the following steps are taken:

(i) A subsample $\left(S_{\text {present }}^{i}\right)$ of life cycle maximum intensity values of size $n$ corresponding to the present climate is generated by sampling $n$ times with replacement from the collection of $n_{\text {present }}$ values associated with the seeded cases of the present climate.

(ii) A subsample $\left(S_{\text {future }}^{i}\right)$ of life cycle maximum intensity values of size $n$ corresponding to the future climate is generated by sampling $n$ times with replacement from the collection of $n_{\text {future }}$ values associated with the seeded cases of the future climate.

(iii) The average maximum intensity, $\theta_{\text {present }}^{i}\left(\theta_{\text {future }}^{i}\right)$, for the subsample $S_{\text {present }}^{i}$ (subsample $S_{\text {future }}^{i}$ ) is determined.

2) The proportion of the $M$ random trials in which $\theta_{\text {future }}^{i}>\theta_{\text {present }}^{i}$ is determined. Following standard bootstrap practice, this proportion is taken to be the significance level of the difference between the average maximum intensity in the future-climate and present-day environments.

Note in the subsampling steps 1(i) and 1(ii) that the likelihood of drawing a case from a given year is constrained to be the same for all years. Thus, low-activity years with fewer cases and weaker storms are not (on average) less represented in the subsamples than highactivity years with more numerous cases and stronger storms. This is a more conservative subsampling approach as compared to the basic bootstrap approach, where the likelihood of drawing a case from a given year is proportional to the number of cases in that year.

\section{Results}

\section{a. Case studies of seeding}

Two case studies help to illustrate the seeding results (Figs. 7 and 8). Figures $7 \mathrm{a}-\mathrm{c}$ show the 15 seeded simulations for 0000 UTC 10 September 2098 that are generated using the conservative configuration and CCSM4 large-scale environmental conditions. Of the seeds, 5 fail to develop, while the other 10 develop at varying intensification rates and display a wide range of maximum intensities (Figs. 7b,c). Most of the resulting TCs track to the northwest and then either recurve or make landfall and dissipate (Fig. 7a). Two storms (highlighted by the red arrows in Fig. 7a) achieve roughly 145-kt MWS prior to landfall. One crosses the South China Sea and makes landfall in China, while the other recurves and makes landfall in southern Japan.

For comparison, Fig. 8 shows the 15 seeded simulations for 0000 UTC 5 October 2096 that are generated using the conservative configuration and CM5A largescale environmental conditions. As with Fig. 7, there is discernible spread in terms of the evolution of the 15 seeds. In this case, 12 of the seeds undergo further development, while 3 fail to meaningfully intensify (Figs. 8b,c). One of the storms ultimately achieves an MWS of $136 \mathrm{kt}$ and minimum central pressure (MCP) of less than $900 \mathrm{hPa}$, although its track remains in the open ocean for its entire life cycle (Figs. 8a-c; highlighted by the red arrow). Most of the storm tracks show strong recurvature over the course of the TC life cycle (Fig. 8a).

\section{b. Summary scatterplots}

Figures 9 and 10 present summary scatterplots of MCP versus MWS and MCP versus latitude for both the conservative and aggressive scenarios. The data points are taken at 24-h intervals from each and every simulated TC life cycle. Considering first the scatterplots of MCP versus MWS (Fig. 9), note that the closer the data point is to the lower-right corner of the plot, the more intense the TC is. For the conservative case, the CCSM4 mean MWS for the future is little changed from the present, but the 90th percentile of MWS and both the mean and 90th percentile of MCP are all discernably stronger for the future (Fig. 9a). The CM5A mean and 90th percentile of both MWS and MCP are all stronger for the future (Fig. 9c). In particular, the 90th percentile of MWS (MCP) is roughly $20 \mathrm{kt}(20 \mathrm{hPa})$ greater, and the data points for the CM5A future simulations extend discernably further into the lower-right corner of the plot. Both the CCSM4 and CM5A simulations yield maximum values of MWS and MCP of roughly 150-160 kt and 875$880 \mathrm{hPa}$. For the aggressive case (Figs. 9b,d), the same general conclusions apply, but the differentiation between future and present simulations is more marked. Both the CCSM4 and CM5A future simulations yield stronger mean and 90th percentiles for MWS and MCP, and the data points for the future simulations extend much further into the lower-right corner. Maximum values of MWS and MCP for the future simulations are roughly $170-180 \mathrm{kt}$ and $845-850 \mathrm{hPa}$.

Looking at the scatterplots of MCP versus latitude (Fig. 10), one finds a contrast between the CCSM4 and 

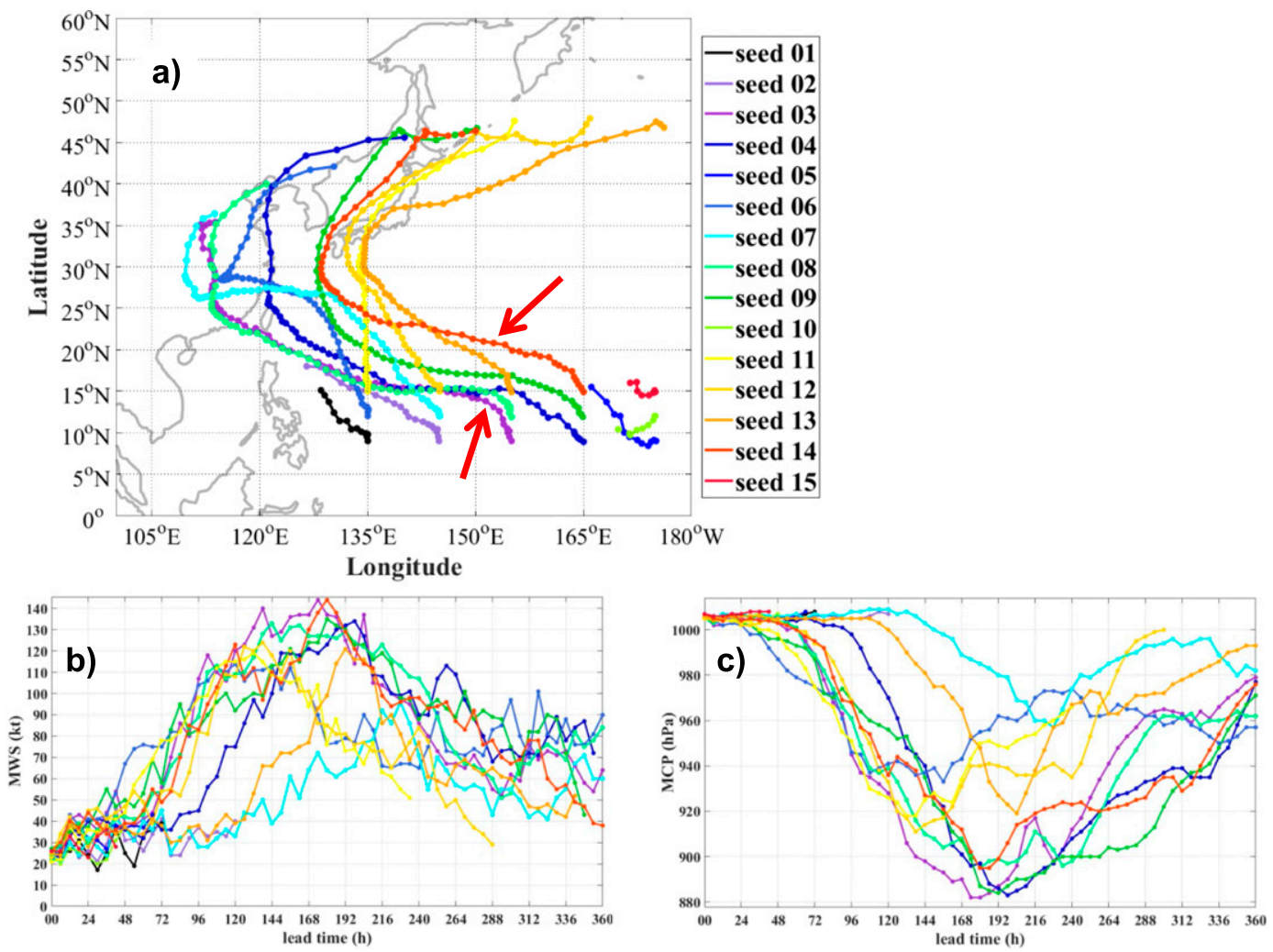

FIG. 7. (a) Life cycle tracks of the 15 vortices seeded 0000 UTC 10 Sep 2098 using the conservative configuration and CCSM4 environmental data. Filled circles show storm location at 6-h intervals. (b) MWS of the 15 vortices seeded in (a). Filled circles are every $6 \mathrm{~h}$. (c) MCP of the 15 vortices seeded in (a).

CM5A results in terms of the mean and 90th percentile of latitude. Namely, for both the conservative and aggressive scenarios, the mean and 90th percentile of latitude for the CCSM4 data points do not differ much between the future and present (Figs. 10a,b), while the mean and 90th percentile of latitude for the CM5A data points are discernably farther north (Figs. 10c,d). However, the CCSM4 and CM5A results do have two interpretations in common. First, TCs of a given intensity tend to be found farther north in the future years than in present day. For example, TCs of roughly 920 930-hPa MCP are seen as far north as $45^{\circ}-46^{\circ} \mathrm{N}$ latitude in the future years, whereas in the present-day TCs of this intensity are found no farther north than $35^{\circ}-38^{\circ} \mathrm{N}$. A second, alternative interpretation is that the maximum TC intensity that is observed for a given latitude tends to be higher for the future-climate years than in the present day. At $45^{\circ} \mathrm{N}$, for instance, the maximum TC intensity that is observed in the conservative scenario is 919-hPa MCP for the future and 933-hPa MCP for the present day.

To examine the aggregate distribution of TC tracks and the relationship between track and intensity, Fig. 11 presents all of the tracks simulated for several selected years of the CCSM4 and CM5A data using the aggressive configuration. Each track is color coded at 24-h intervals based on the maximum TC wind speed at the time. Looking at Fig. 11a that shows the tracks simulated using year 2098 of the CCSM4 data, it is apparent that a substantial number of the TC life cycles attain a category 4 or 5 intensity on the Saffir-Simpson hurricane wind scale (113-136 or $\geq 137 \mathrm{kt}$, respectively; Simpson and Riehl 1981), consistent with the corresponding maximum intensity plots of Fig. 12 and with the fact that both the genesis index (Emanuel and Nolan 2004) and the potential intensity are much higher across the WestPac in 2098 than in the other years (not shown), mainly as a result of anomalously warm SSTs (see Fig. 2b). The highest intensities tend to occur between the latitudes of $20^{\circ}$ and $35^{\circ} \mathrm{N}$, and there is indication that a considerable number of the TCs that reach high intensity are able to maintain this high intensity all the way to landfall in Taiwan, the Korean Peninsula, and Japan. This latter point will be revisited in section $3 \mathrm{f}$ below. There does not appear to be any one predominant track structure, as some of the storms recurve, while a substantial fraction moves generally westward. For comparison, Fig. 11b shows the tracks simulated using year 2099 of 

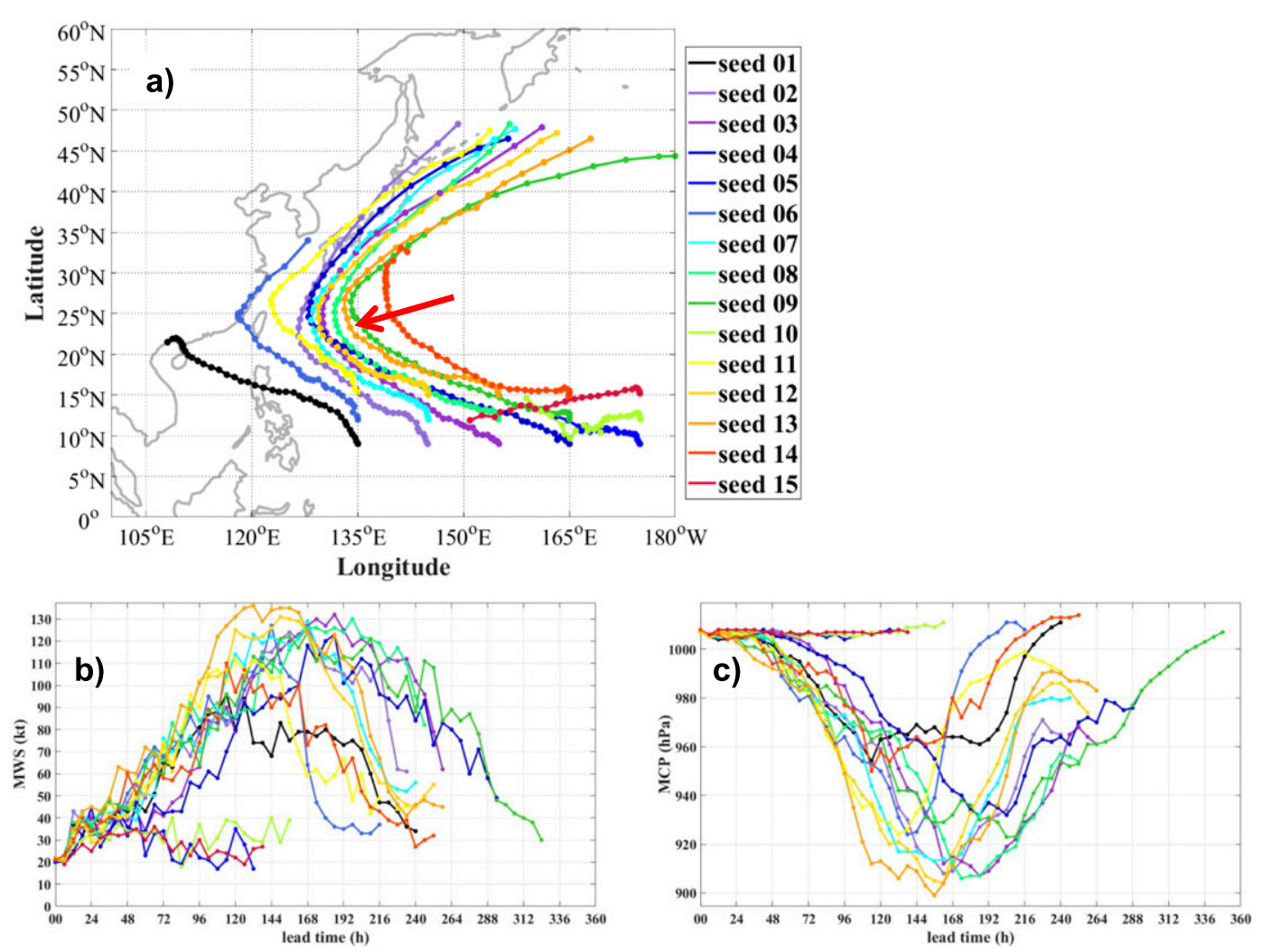

FIG. 8. As in Fig. 7, but for the 15 vortices seeded 0000 UTC 5 Oct 2096 using the conservative configuration and CM5A environmental data.

the CCSM4 data. The presence of interannual variability is very clear when contrasting Fig. 11a and Fig. 11b, with Fig. 11b showing a much smaller proportion of category 4 and 5 storms than Fig. 11a. Also, Fig. 11b has more instances of TCs that move westward without recurvature. As with Fig. 11a, there is no predominant track structure. Finally, Fig. 11c shows those tracks simulated using year 2097 of the CM5A data. The tendency for the CM5A storms to strongly recurve, suggested above in Fig. 8a, is plainly evident in Fig. 11c. There is some indication that this tendency may be due to the climatological high pressure zone over the central Pacific Ocean being somewhat weaker in the CM5A data as compared to the CCSM4 data, but the underlying phenomenology was not investigated in detail for this study. Nevertheless, the contrast in track structure between the CCSM4 and CM5A simulations is consistent with the variability in TC track across CMIP5 models noted by Wang and $\mathrm{Wu}$ (2015). Also, like in Fig. 11a for the year 2098 CCSM4 cases, the most intense storms tend to occur in the $20^{\circ}-35^{\circ} \mathrm{N}$ latitude band, and a number of these strong storms make landfall in the Korean Peninsula and Japan.

\section{c. Life cycle maximum intensity and latitude of maximum intensity}

Results for average life cycle maximum intensity are presented in Fig. 12, based on those cases where the TC intensity exceeds $96 \mathrm{kt}$ (i.e., achieves category 3 intensity or greater). Considering the results as a function of year (Figs. 12a,b), one finds fairly strong indication for both the conservative and aggressive configurations that the average life cycle maximum intensity is apt to be greater in the future years. This indication holds even if one disaggregates the simulations into 3-yr subsets based on climate model (CCSM4/CM5A). In fact, all future years save for CCSM4 year 2099 have greater average life cycle maximum intensity than any of the present years. The CM5A future-year results, in particular, are substantially greater than their present-day counterparts. Also, recalling from section $2 b(4)$ that the real-world cases are counterparts to the seeded simulations with the aggressive configuration, it is worth comparing in Fig. 12b the real-world results (represented by the black bar) with the seeded-simulation results. One finds that the present-year seeded-simulation average intensities are generally comparable to the average intensity of the 

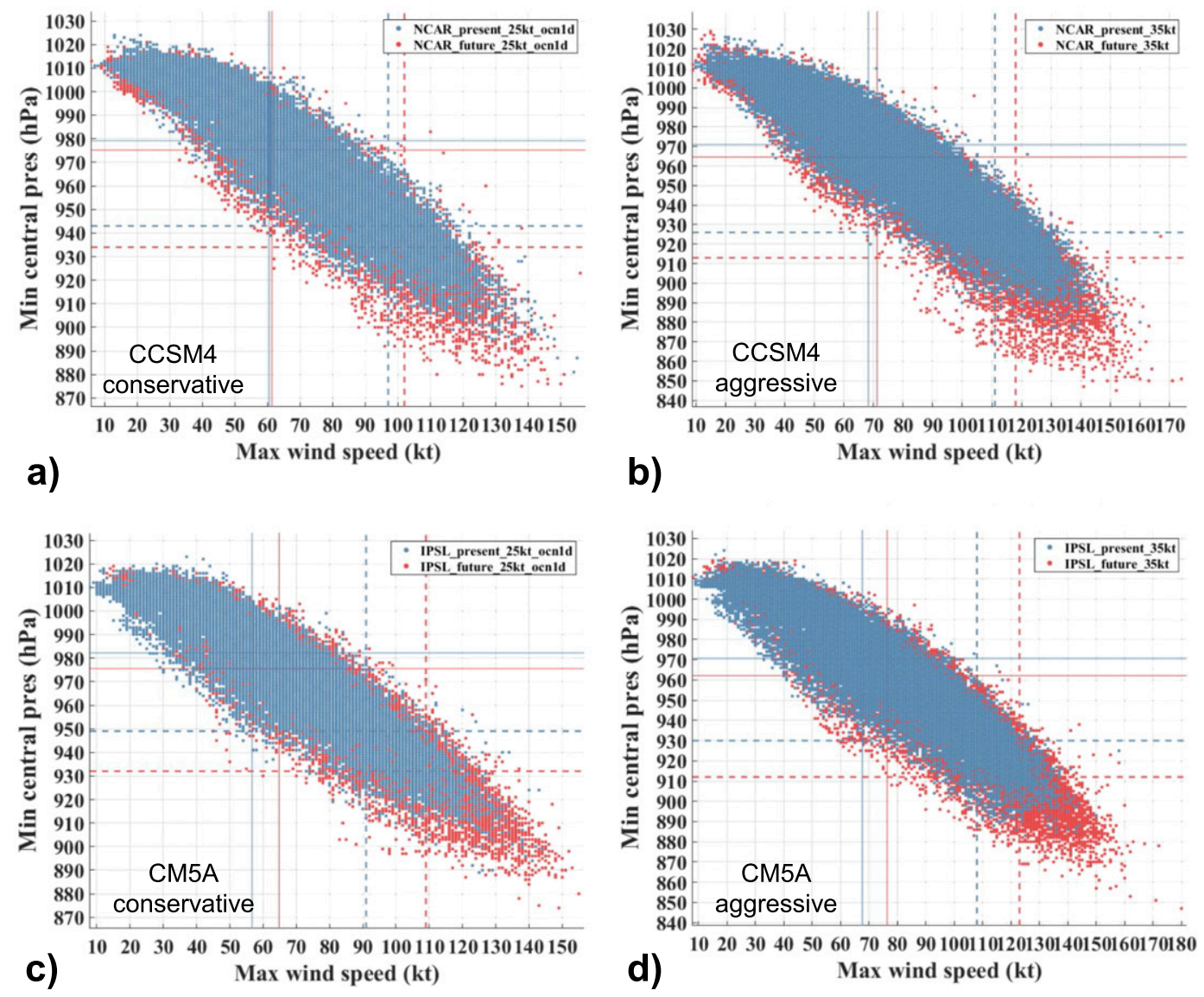

FIG. 9. (a) MCP vs MWS for seeded simulations using the conservative configuration and CCSM4 data for the present years (blue) and future years (red). Data are every six hours over the life cycle. Solid (dashed) lines denote the associated average (90th percentile) values for the simulations. (b) As in (a), but for simulations using the aggressive configuration. (c) As in (a), but using CM5A data. (d) As in (a), but using the aggressive configuration and CM5A data.

real-world cases (as might be expected), whereas the future-year seeded-simulation average intensities all substantially exceed the average intensity of the realworld cases. Thus, the future-year average intensities prove greater than those of present day in two different comparisons (one using the present-day seeded simulations and one using the real-world cases).

Figures $12 \mathrm{c}$ and $12 \mathrm{~d}$ summarize the significance test results associated with the life cycle maximum intensity for the case where $M=1 \times 10^{6}$ and $n=100$. There is essentially no overlap between the future-climate histogram and the present-day histogram and there is clear separation between the mean values of the histograms. These results translate into a significance level of $>99 \%$ for the difference between the average maximum intensity in the future-climate and present-day environments. For the conservative configuration, the expected increase in the average maximum intensity in the future is $\sim 7 \mathrm{kt}$, while for the aggressive configuration, the expected increase is $\sim 10 \mathrm{kt}$. Similarly, strong significance results are obtained in the case where the intensity threshold is taken to be $64 \mathrm{kt}$ and $n=100$ (i.e., when only cases where the TC reaches category 1 or greater are considered; not shown). Thus, the evidence strongly suggests that the life cycle maximum intensity will be greater, on average, in the future-climate environments.

Results for the latitude of life cycle maximum intensity are shown in Fig. 13. Here the results are not as clear as those for the average maximum intensity. Specifically, if the simulations are disaggregated into 3-yr subsets based on climate model (CCSM4/CM5A), one finds that the CM5A simulations consistently suggest an increase in the average latitude of life cycle maximum intensity in the future, both for the conservative and aggressive configurations (Figs. 13a,b). Meanwhile, the CCSM4 simulations are ambivalent, with the year 2098 simulations suggesting an increase in average latitude and the other years suggesting a decrease or little 


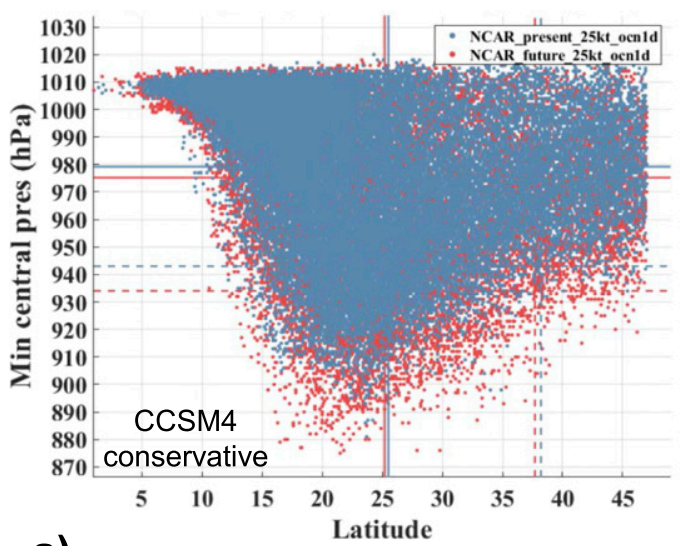

a)

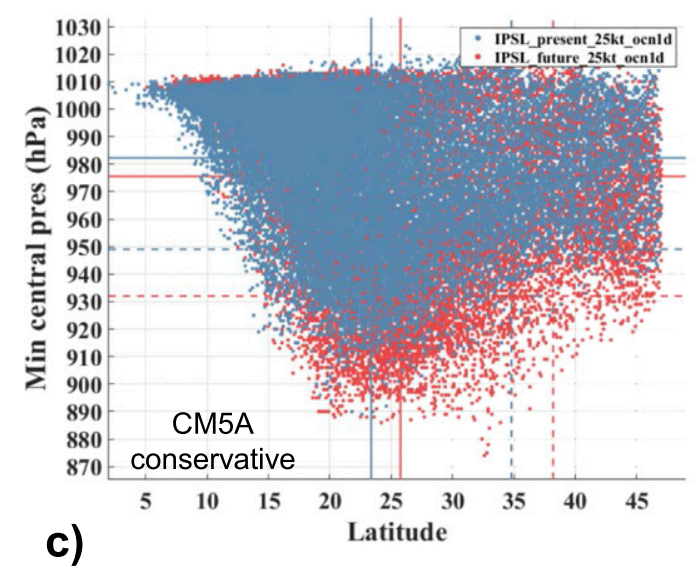

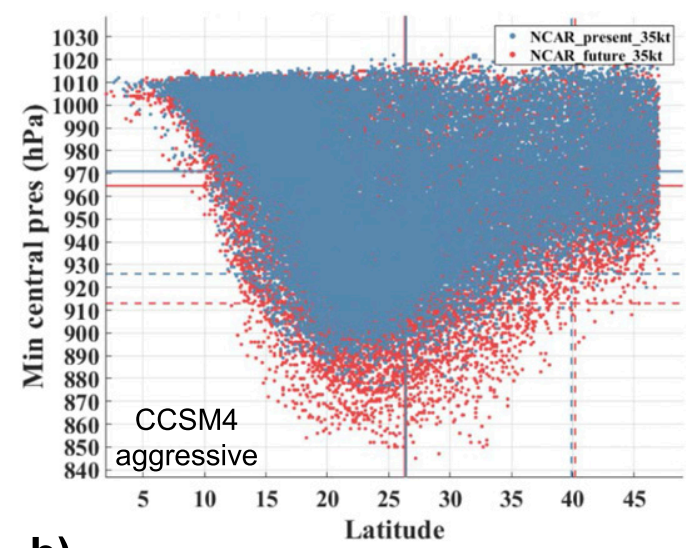

b)

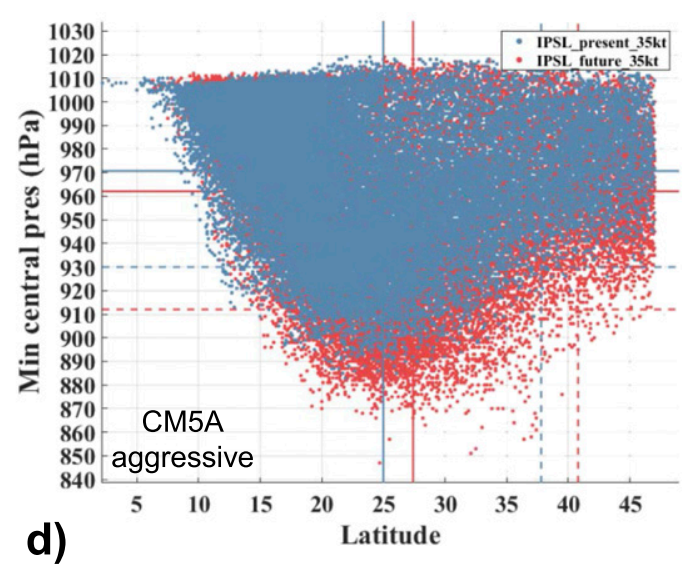

FIG. 10. (a) MCP vs latitude for seeded simulations using the conservative configuration and CCSM4 data for the present years (blue) and future years (red). Data are every six hours over the life cycle. Solid (dashed) lines denote the associated average (90th percentile) values for the simulations. (b) As in (a), but for simulations using the aggressive configuration. (c) As in (a), but using CM5A data. (d) As in (a), but using the aggressive configuration and CM5A data.

change, depending on the choice of conservative or aggressive configuration. These qualitative observations are echoed in the significance test results. For instance, Fig. $13 \mathrm{c}$ shows the significance results for the conservative configuration when the CCSM4 and CM5A years are treated as an aggregate 6-yr set and using the same values of $M$ and $n$ as in Figs. 12c,d. There is substantial overlap of the histograms, and the significance level for the difference in average latitude is $<99 \%$. By contrast, Fig. 13d shows the significance results for the conservative configuration when comparing only the three CM5A future years against the three CM5A present years. Here there is a clear separation of the histograms of average latitude for the future and present, and the expected average latitude is $\sim 1.5^{\circ}$ farther north in the future. Analogous results apply for the aggressive configuration (not shown). Thus, while the simulation results overall suggest a northward shift of the average latitude of the life cycle maximum intensity in the future climates, there is too much interannual variability across the CCSM4 years to make a formal statement of significance.

The longitude of life cycle maximum intensity was also investigated in view of the fact that some previous studies have reported a general eastward shift in TC tracks in future climates (e.g., Murakami et al. 2011; Yokoi et al. 2013). Considering the average longitude of maximum intensity, no formally significant difference is found between present and future TCs (not shown). This is due largely to there being substantial interannual variability across both the present and future years. If one considers the 90th percentile of the longitude of maximum intensity (not shown), then the results exhibit some model dependence. Specifically, for the seeded simulations based on CCSM4 data, the 90th percentile of the longitude of life cycle maximum intensity is detectably farther eastward for the future TCs. Meanwhile, for the seeded simulations based on CM5A data, there is 

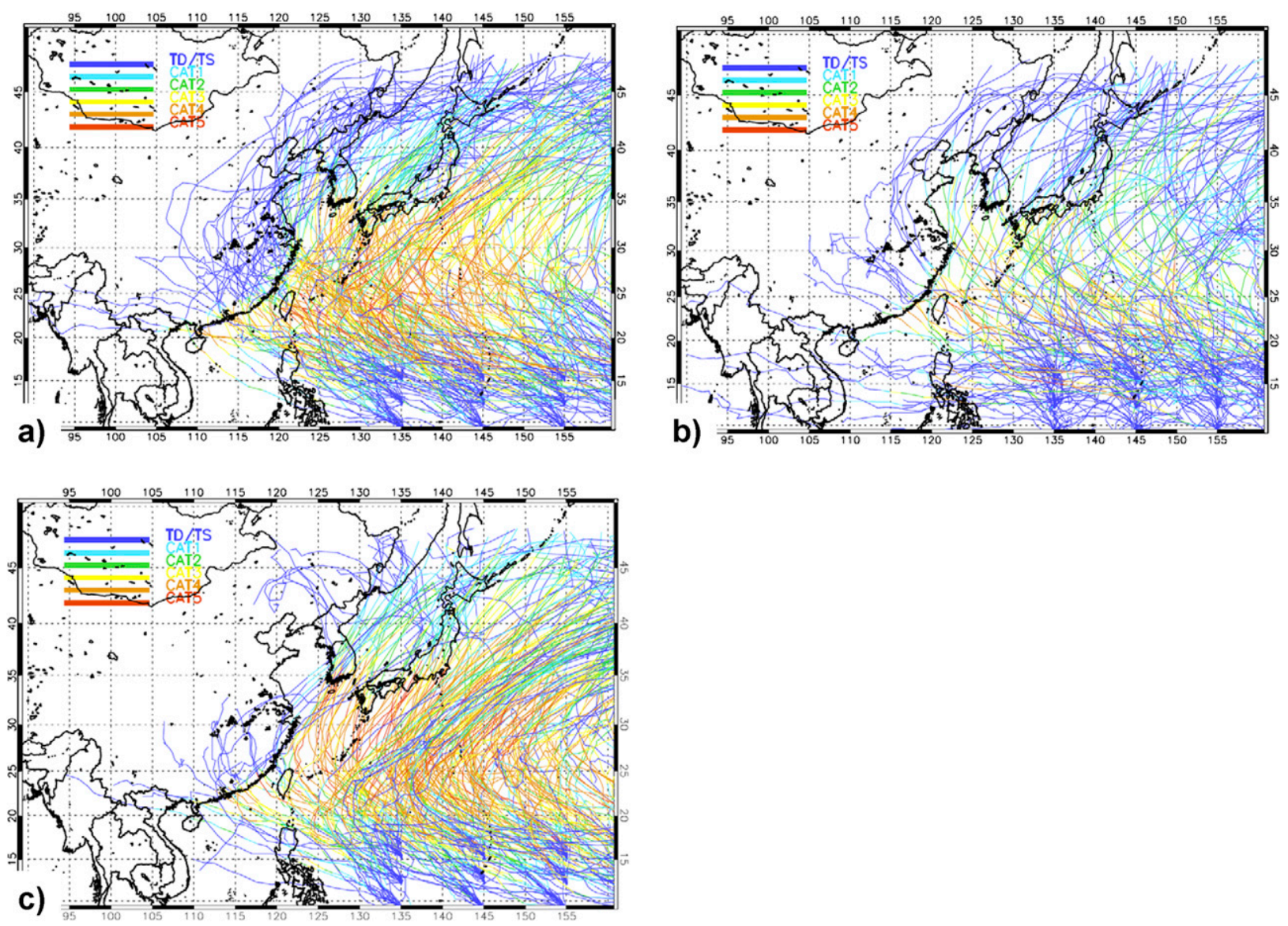

FIG. 11. (a) Tracks from all 280 seeded simulations for the months August-October of the year 2098 using the aggressive configuration and CCSM4 environmental data. Each track is color coded at 24-h intervals based on storm intensity according to the legend at the upper left. (b) As in (a), but for the year 2099. (c) As in (a), but for the year 2097 using the aggressive configuration and CM5A environmental data.

no significant difference between the 90th percentile of longitude for the present and future TCs.

\section{d. Intensification rate}

Figure 14 shows frequency histograms of intensification rate for both the present-day and future-climate periods, where the rate is defined as the change in maximum wind speed over a 24 -h interval. For both the conservative and aggressive configurations, there is some suggestion that the distribution of intensification rate for the future simulations has more weight in the tails or, equivalently, that the distribution for the future simulations has more variance. This is most apparent for the CM5A distributions (Figs. 14c,d). The CM5A distributions for the future also exhibit larger maximum values than those for present day, on both the positive and negative tails of the distribution. It is possible that the COAMPS-TC model places an artificial constraint on the intensification rate, since historically even highresolution NWP models have struggled to replicate observed TC intensification rates (e.g., Gall et al. 2013). Thus, Fig. 14 may underestimate the differences in the distributions of intensification rate between the presentday and future environments.

\section{e. Intensity over time}

Another important aspect of the TC life cycle is the relationship between intensity and time. For instance, it is one thing if the TCs in a given environment are characterized by high intensity but short duration and another if the TCs are characterized by high intensity and long duration. A common scalar measure of the relationship between TC intensity and duration is the average number of hours per life cycle that a TC maintains an intensity at or above a specified threshold.

Figures $15 \mathrm{a}$ and $15 \mathrm{~b}$ show this measure $\tau$ for those storms that exceed the 96-kt intensity threshold. This choice of threshold highlights storms of category 3 intensity or higher that are capable of doing major damage. Considering the conservative configuration (Fig. 15a), 

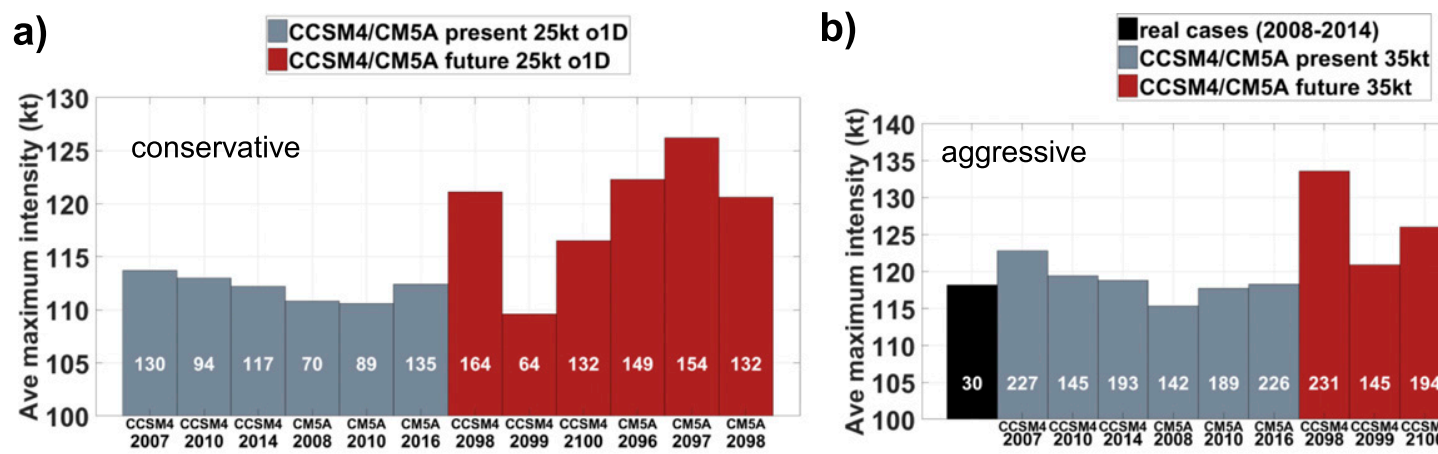

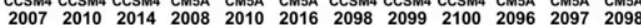

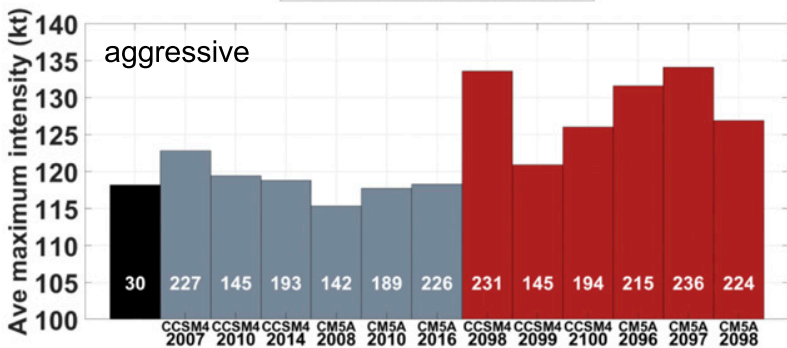

c)

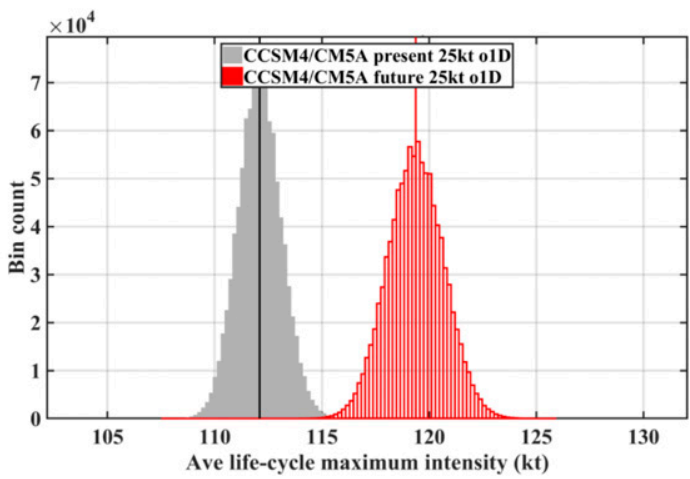

d)

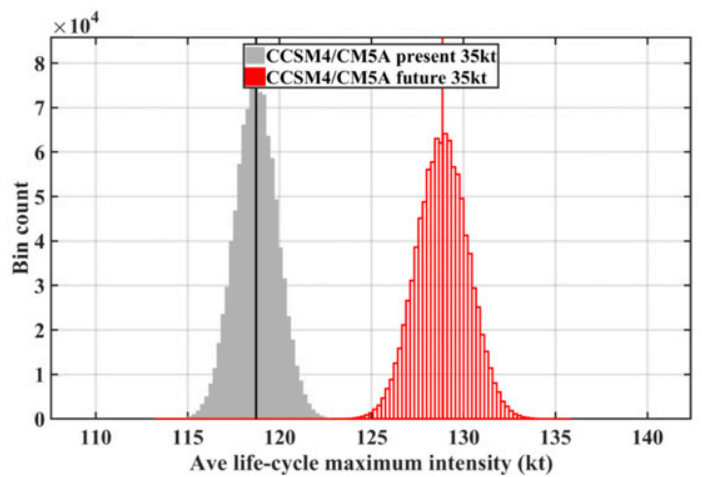

FIG. 12. (a) For the conservative configuration, the average life cycle maximum intensity for those TCs whose MWS is $>96 \mathrm{kt}$ at some point during the life cycle. The gray (red) bars represent the seeded simulations using climate-model environments for the present (future) years. The white number superimposed on each color bar indicates the number of cases that meet the criterion that the MWS is $>96 \mathrm{kt}$ at some point during the life cycle. (b) As in (a), but for the aggressive configuration. The black bar represents the simulations for the realworld cases. (c) Significance test results corresponding to (a). The gray and red histograms represent the sampling distributions of $\theta_{\text {present }}$ and $\theta_{\text {future }}$, respectively. The bold vertical colored lines indicate the mean values of the respective sampling distributions. See text for further details. (d) Significance test results corresponding to (b).

one finds that the $\tau$ values of all but one of the future years exceed the largest $\tau$ value of the present years (that for CCSM4 year 2010). Further, the largest $\tau$ value of the future years (that of CM5A year 2097) exceeds the largest $\tau$ value of the present years by $25 \mathrm{~h}$, or more than one day. Comparable results are seen for the aggressive configuration (Fig. 15b). Note in Fig. 15b that, similar to the results for the life cycle maximum intensity, the $\tau$ values of the future years exceed not only the values of the present years but also the $\tau$ value of the real-world cases.

The statistical significance of these results was tested using the method of section $2 \mathrm{~b}(6)$ with $n=100$ and $M=$ $1 \times 10^{6}$, and Fig. $15 \mathrm{c}$ shows the corresponding sampling distributions of $\theta_{\text {present }}$ and $\theta_{\text {future }}$ for the conservative configuration. The distribution of $\theta_{\text {future }}$ is clearly shifted toward higher values in comparison to that of $\theta_{\text {present }}$, and the corresponding significance level of the difference between $\theta_{\text {present }}$ and $\theta_{\text {future }}$ is $>99 \%$. The expected $\tau$ for the future years (i.e., the mean of the sampling distribution of $\left.\theta_{\text {future }}\right)$ is $19 \mathrm{~h}$ greater than the expected $\tau$ for the present years. The sampling distributions for the aggressive configuration (Fig. 15d) yield similar observations.

As with the results for life cycle maximum intensity (Fig. 13), the CCSM4 year 2099 stands out for its low average value in comparison to the other future years. However, even with the low $\tau$ value of CCSM4 year 2099 the CCSM4 future years still yield statistically significantly greater average $\tau$ than the CCSM4 present years (not shown).

Additionally, analogous results are obtained if one includes TCs that do not exceed the 96-kt threshold in the calculation of $\tau$ (not shown). Thus, even with the interannual variability of the CCSM4 results, the overall indication is that the future extreme-climate environments allow TCs to maintain high intensity for longer duration than is typical in the present-day environment. The physical reason that the future-climate simulations favor larger $\tau$ is not entirely clear. One speculation is that the future-climate environments allow a storm to reach high intensity farther out to sea than in present day, thereby increasing the amount of time between 
a)

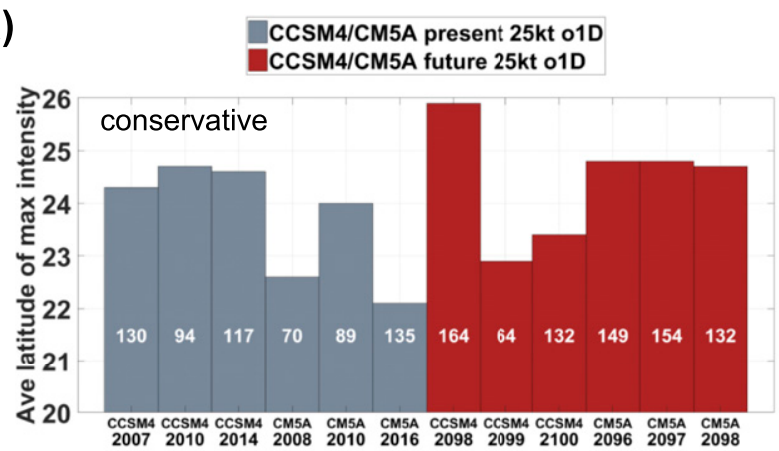

c)

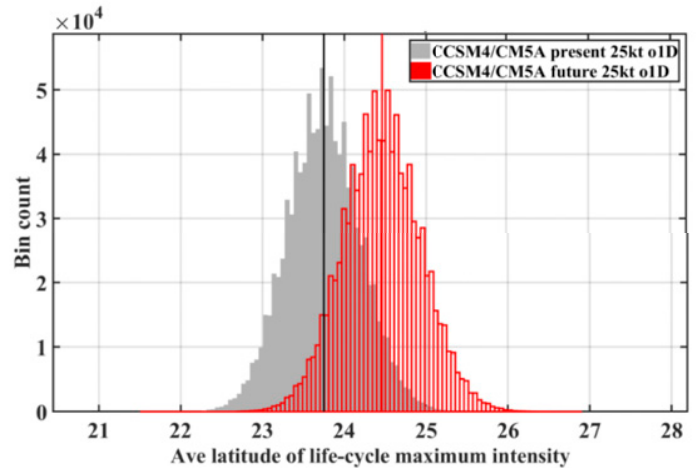

b)
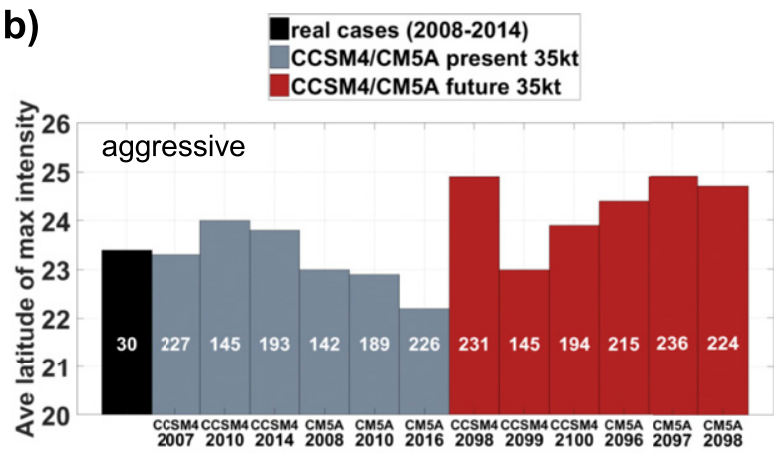

d)

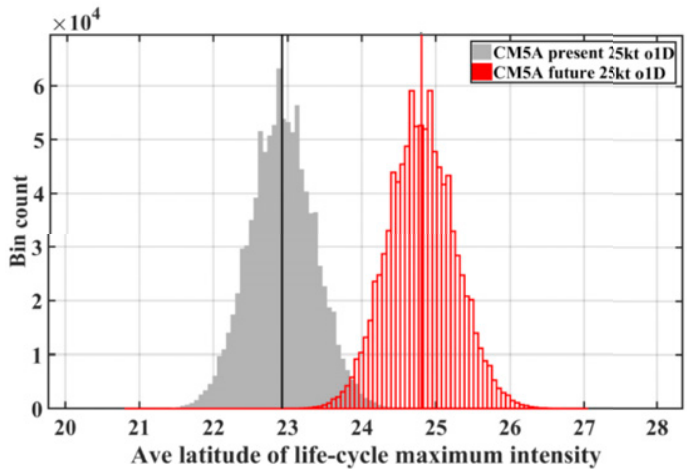

FIG. 13. (a) For the conservative configuration, the average latitude of the life cycle maximum intensity for those TCs whose MWS is $>96 \mathrm{kt}$ at some point during the life cycle. Otherwise, as in Fig. 12a. (b) As in (a), but for the aggressive configuration. The black bar represents the simulations for the real-world cases. (c) Significance test results corresponding to (a). The gray and red histograms represent the sampling distributions of $\theta_{\text {present }}$ and $\theta_{\text {future }}$, respectively. The bold vertical colored lines indicate the mean values of the respective sampling distributions. See text for further details. (d) Significance test results corresponding to (a), but considering only the CM5A present years vs the CM5A future years.

when the storm first reaches high intensity and when it makes landfall and weakens.

\section{f. Intensity at first landfall}

Tropical cyclones in the WestPac are of particular importance given the concentrations of population and infrastructure in the coastal regions of Asia that are exposed to the risk of TC-landfall events. Thus, the intensity of WestPac TCs at their first major landfall is just as important of a metric as the maximum intensity attained by these TCs over their life cycle, because the maximum intensity may tend to be reached in the open ocean away from population centers and critical infrastructure. Figure 16 presents both the average and maximum intensity at first landfall for those cases that meet the criterion that the MWS at landfall is $>64 \mathrm{kt}$. The first landfall is determined based upon a $1^{\circ} \times 1^{\circ}$ land mask. Comparing the six present and future years on aggregate, there is a general tendency for the average and maximum wind speeds at landfall to be greater in the future-climate years than in present day. For instance, under both the conservative and aggressive configurations, five of the six future years yield greater average landfall intensity than the largest average landfall intensity value seen from the present years (i.e., the value for CCSM4 year 2010). Under the conservative configuration, the largest average wind speed and the largest maximum wind speed are both observed in the year 2097 of the CM5A simulations, with the largest maximum wind speed of 148-kt associated with a TC that comes ashore in the Nagasaki, Japan, region. Similar results are seen for the average and minimum MCP at landfall (not shown). The most extreme minimum MCP at first landfall is $889 \mathrm{hPa}$, associated with a TC in year 2097 of the CM5A simulations that strikes the southern coast of the Korean Peninsula.

Applying the significance test of section $2 b(6)$ to the results of Fig. 16a with $n=100$ and $M=1 \times 10^{6}$ yields the sampling distributions of $\theta_{\text {present }}$ and $\theta_{\text {future }}$ shown in Fig. 16c. There is clearly some separation between the future-climate sampling distribution and the presentclimate distribution, and the corresponding significance level for the difference between the average landfall intensity in the future and the present day is greater than $99 \%$. Comparing the means of the two sampling distributions, the expected average intensity at first landfall in 

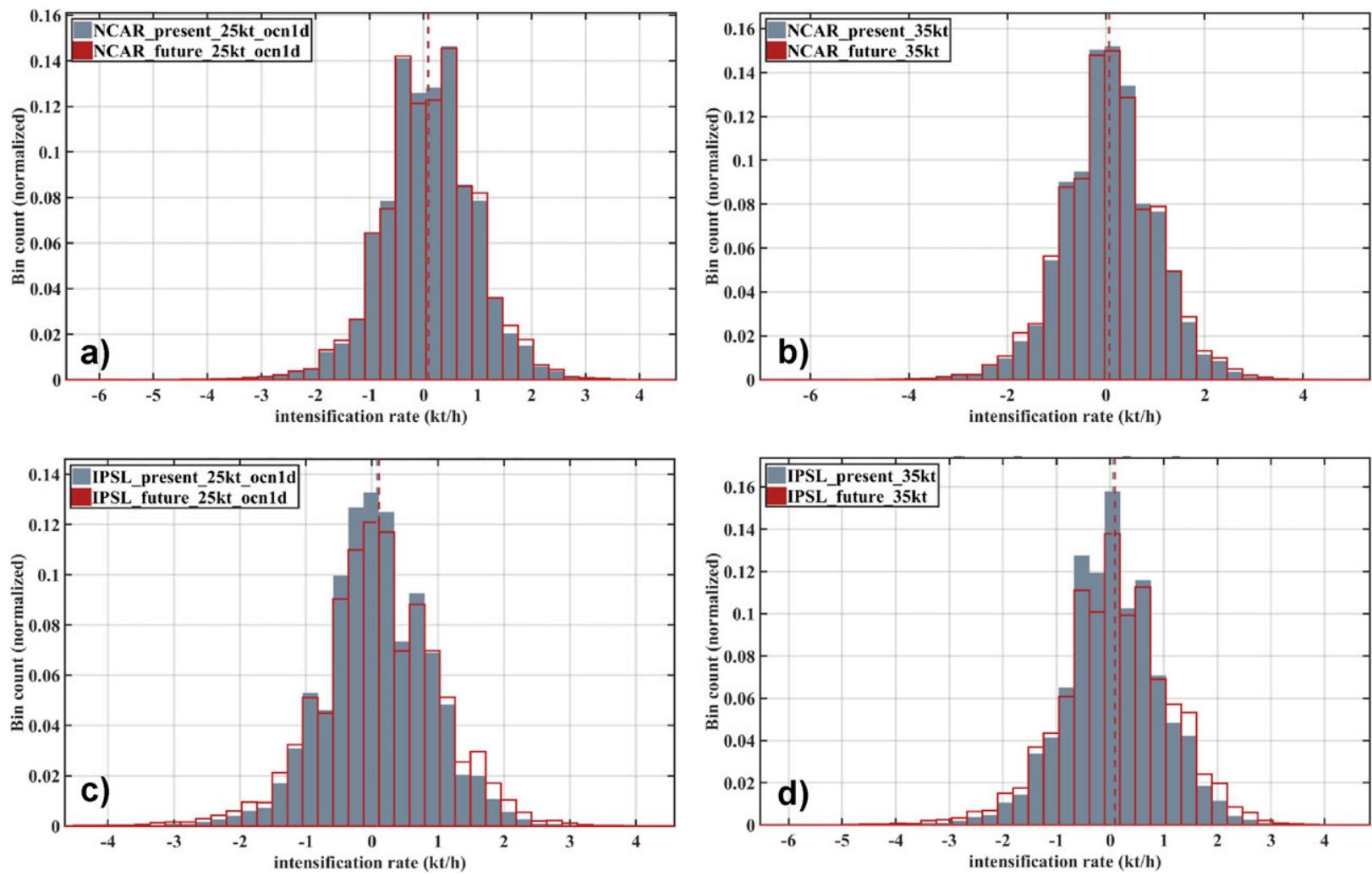

FIG. 14. (a) Distribution of intensification rate for seeded simulations using the conservative configuration and CCSM4 data for the present years (gray) and future years (red). (b) As in (a), but for simulations using the aggressive configuration. (c) As in (a), but using CM5A data. (d) As in (a), but using the aggressive configuration and CM5A data.

the future years is roughly $6 \mathrm{kt}$ greater than in the present. Significance test results for the aggressive configuration are similar and suggest an expected future increase in average landfall intensity of roughly $10 \mathrm{kt}$ (not shown).

There is not much interannual variability in the results for the future years, but year 2099 of the CCSM4 simulations does stand out by yielding substantially fewer landfall cases, and substantially lower average intensity at first landfall, than any of the other future years (and even some of the present years). Largely because of year 2099, the results do have an element of model dependency. In particular, if one compares the three CCSM4 future years against the three CCSM4 present years, there continues to be a suggestion of a future increase in average intensity at first landfall, but the expected increase is smaller than that of Fig. 16c ( $\sim 3 \mathrm{kt}$ for the conservative configuration) and not formally statistically significant at a $90 \%$ level (not shown). By the same token, comparing the CM5A future years against the CM5A present years, the future increase in average intensity at first landfall is much larger than that of Fig. 16c. Specifically, for the conservative configuration the expected future increase is roughly $11 \mathrm{kt}$ (Fig. 16d).

Nonetheless, when the six present and future years are viewed in aggregate, the results of Fig. 16 strongly suggest that the extreme future climates could support the landfall of noticeably more intense storms. Based on analysis of the terms of the genesis index and potential intensity in the future climates (not shown), this circumstance is likely attributable in large part to the presence in some of the future-climate years of a warmer and more expansive WestPac SST warm pool and to a general reduction in the environmental vertical wind shear over Japan and the Korean Peninsula. In year 2098 of the CCSM4 data, in particular, the WestPac SSTs during the summer and fall months are $2-4 \mathrm{~K}$ warmer than present day even as far north as Japan.

When considering the above landfall results, it should be emphasized that they are germane to TCs that originate in the open ocean of the WestPac region. The investigation of TCs that originate in the South China Sea, another important source region of landfalling TCs, is left for future work. It may well be 
a)

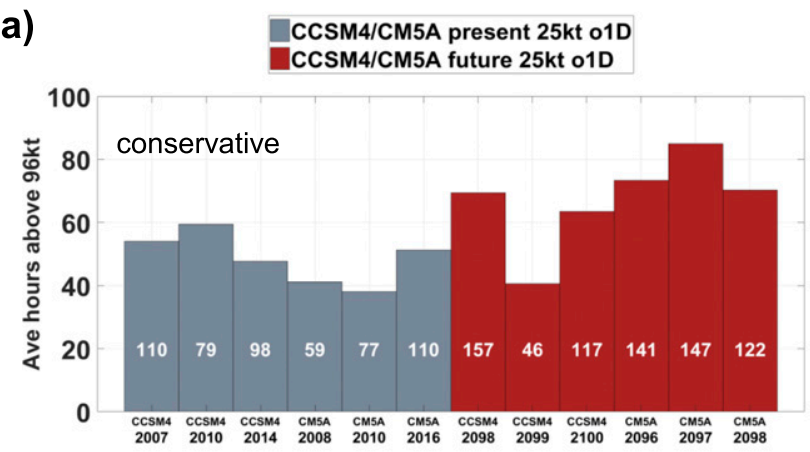

c)

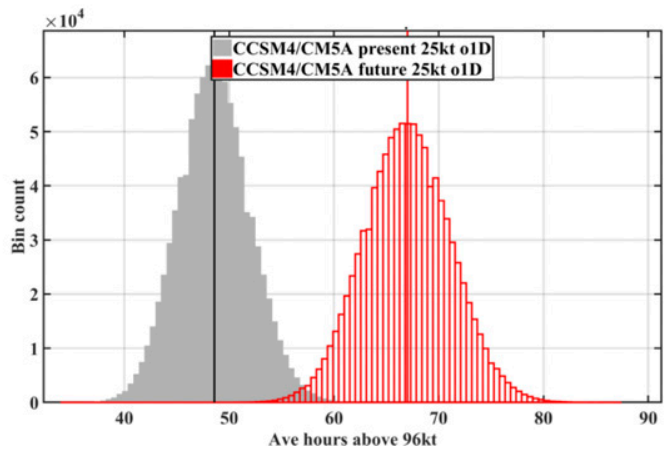

b)

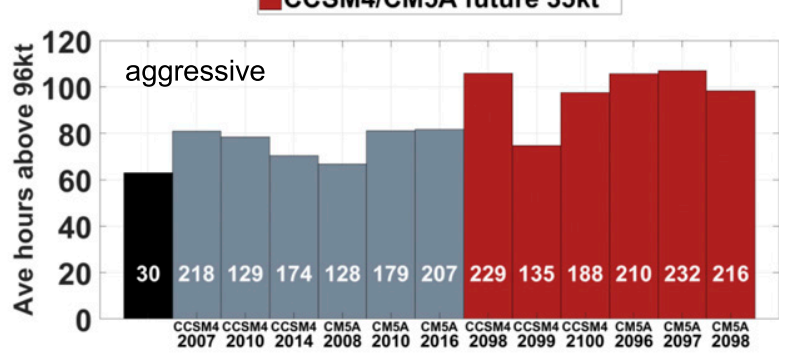

d)

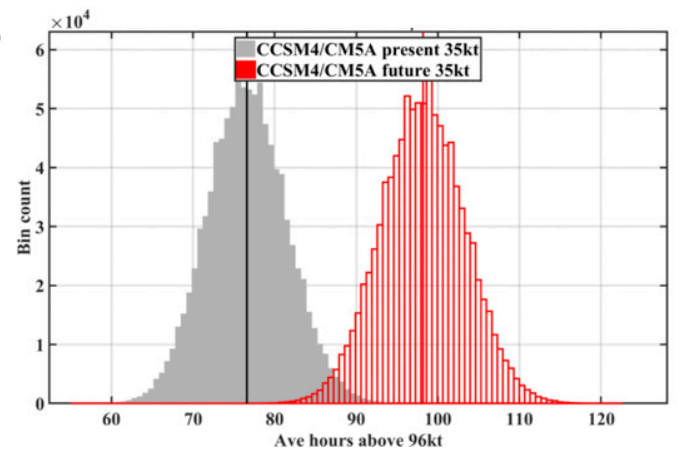

FIG. 15. (a) For the conservative configuration, the average number of hours per life cycle that a TC simulation maintains an MWS $>96 \mathrm{kt}$, considering only those TCs whose MWS is $>96 \mathrm{kt}$ at some point during the life cycle. The gray (red) bars represent the seeded simulations using climate-model environments for the present (future) years. The white number superimposed on each color bar indicates the number of cases that meet the criterion that the MWS is $>96 \mathrm{kt}$ for at least one contiguous 6-h interval during the life cycle. (b) As in (a), but for the aggressive configuration. The black bar represents the simulations for the real-world cases. (c) Significance test results corresponding to (a). The gray and red histograms represent the sampling distributions of $\theta_{\text {present }}$ and $\theta_{\text {future }}$, respectively. The bold vertical colored lines indicate the mean values of the respective sampling distributions. See text for further details. (d) Significance test results corresponding to $(b)$.

that the statistics for South China Sea TCs are distinct from those presented here.

\section{g. Storm size and speed}

Additional TC properties of general interest are the size of the TC [often measured by the radius of 34-kt wind speed (R34)] and the speed at which the storm moves (as measured by the rate of translation of the storm center). The storm size is an important factor in a storm's overall destructive potential (e.g., Sun et al. 2017), while the storm speed is a predictor of stormsurge effects and of cold-wake effects related to mixing in the upper ocean. Figures $17 \mathrm{a}$ and $17 \mathrm{~b}$ show for each year the average of R34 over all cases where the storm intensity as measured by MWS reaches at least $96 \mathrm{kt}$ (category 3 intensity). The indications are that there is a small but discernible increase in R34 for the future years, both for the conservative and aggressive configurations. This indication stands whether one considers the years as 6-yr aggregate sets or one disaggregates them into their respective 3-yr CCSM4/CM5A subsets. Significance tests of the form described in section $2 b(6)$ were applied to the results of Figs. 17a,b and the tests support an increase in R34 in the future years, but at a less stringent significance level of $90 \%$ (Figs. 17c,d). The difference in expected average R34 between the future and present years, calculated as the difference between the means of the two histograms in Fig. 17c or Fig. 17d, is roughly $7-8 \mathrm{n} \mathrm{mi}(1 \mathrm{n} \mathrm{mi}=1.852 \mathrm{~km})$. For the $64-\mathrm{kt}$ threshold (not shown), the results mirror those of the 96-kt threshold, with the difference in expected average R34 being roughly 8-9 $\mathrm{n}$ mi. Thus, the overall indication from Fig. 17 is that the storm size may be slightly larger in the future climates, with major storms (i.e., of at least category 3 intensity) and general storms (of at least category 1 intensity) exhibiting similar size difference. Such an increase in size in future climates is consistent with the results of Sun et al. (2017).

Average storm speed results are shown in Fig. 18 for the 96-kt intensity threshold. Considering either the conservative or aggressive configuration, one finds substantial interannual variability in the average speed across both the present- and future-year sets (Figs. 18a,b). Further, if the present- and future-year sets are 
a)

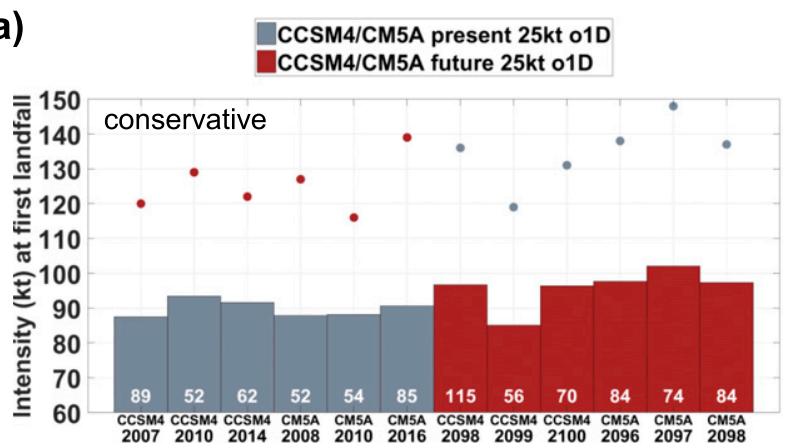

c)

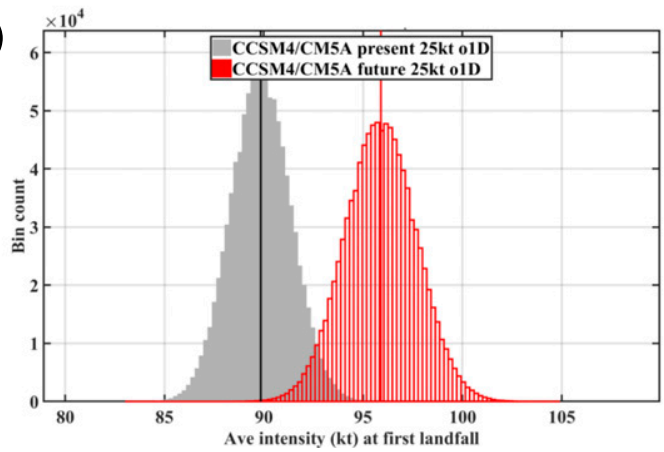

b)

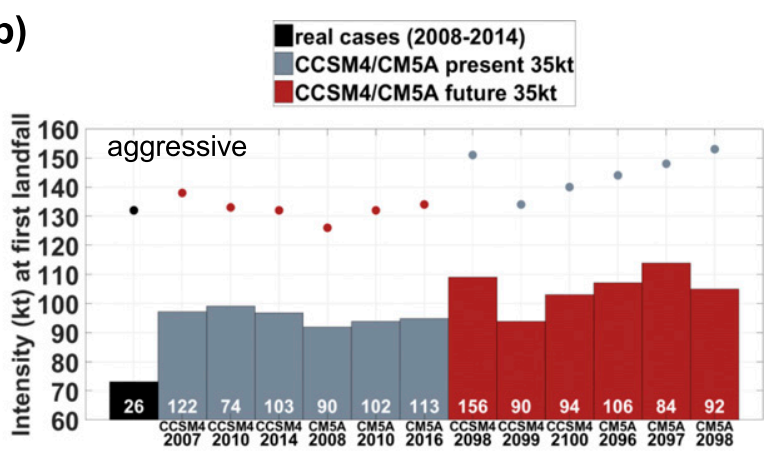

d)

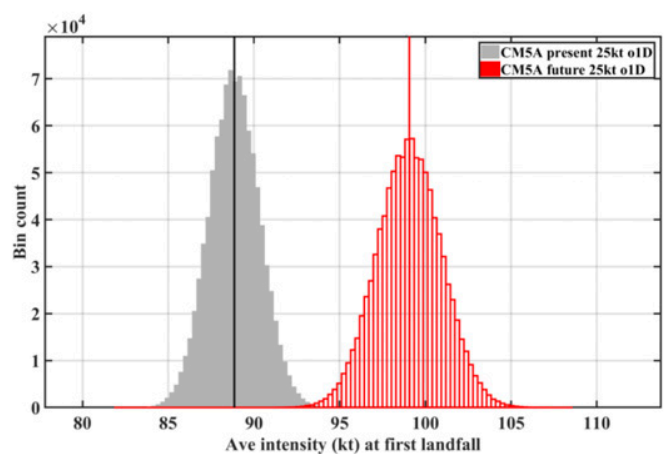

FIG. 16. (a) For the conservative configuration, the average (denoted by the color bars) and the maximum (denoted by the filled circles) landfall intensity, considering only those TCs whose MWS is $>64 \mathrm{kt}$ at landfall. The gray (red) bars represent the seeded simulations using climate-model environments for the present (future) years. The white number superimposed on each color bar indicates the number of cases that meet the criterion that the MWS is $>64 \mathrm{kt}$ at landfall. (b) As in (a), but for the aggressive configuration. The black bar represents the simulations for the real-world cases. (c) Significance test results corresponding to the average landfall intensity in (a). The gray and red histograms represent the sampling distributions of $\theta_{\text {present }}$ and $\theta_{\text {future }}$, respectively. The bold vertical colored lines indicate the mean values of the respective sampling distributions. See text for further details. (d) Significance test results corresponding to the average landfall intensity in (a) but considering only the CM5A present years vs the CM5A future years.

disaggregated into their respective 3-yr CCSM4/CM5A subsets, then the results are ambiguous. Namely, the CCSM4 results suggest that the storms might move slightly slower, overall, in the future as compared to the present, whereas the CM5A results suggest that the storms might move somewhat faster, overall. Significance tests of the form described in section $2 b(6)$ help to better illustrate this contrast (Figs. 18c,d). For the conservative configuration, the CCSM4 results' suggested decrease in average speed is not significant even at an $80 \%$ level (Fig. 18c), while the CM5A results' suggested increase in average speed is significant at a $90 \%$ level (Fig. 18d). The corresponding expected decrease (increase) in average speed indicated by the CCSM4 (CM5A) results is roughly $-0.1(+0.3) \mathrm{m} \mathrm{s}^{-1}$. Average storm speed results for the 64-kt intensity threshold (not shown) are similar in nature, but the distinctions in storm speed are statistically stronger when using the 64-kt threshold. Specifically, for the conservative configuration, the expected decrease (increase) in future average storm speed indicated by the CCSM4 (CM5A) results for the 64-kt threshold is roughly $-0.3(+0.5) \mathrm{m} \mathrm{s}^{-1}$, and these speed differences are significant at a $90 \%$ level or greater. The physical basis of the above speed changes is not investigated for this study, but presumably the changes stem from systematic differences in the largescale environmental flow of the CCSM4 and CM5A models. To summarize, the average storm speed results, like those for the latitude of life cycle maximum intensity (section 3c), are a function of the climate model that was used to define the large-scale environment. Thus, no definitive statement can be made as to whether the TCs are apt to move more slowly or quickly in the future. However, regardless of the choice of climate model, the expected difference in average speed between the future and present tends to be relatively small, and the expected difference is smallest for the strongest storms (those exceeding $96 \mathrm{kt}$ ).

\section{h. Survey of results for supplemental years}

As discussed in section $2 \mathrm{a}(2)$, the selection of years that underlie the main results of the study is somewhat 
a)

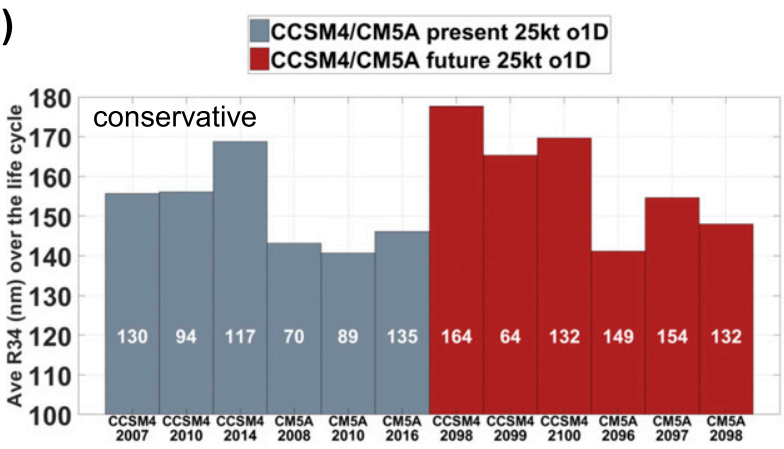

c)

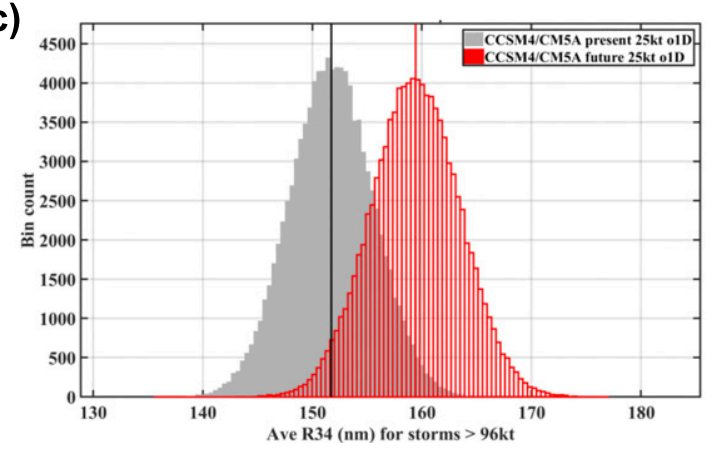

b)

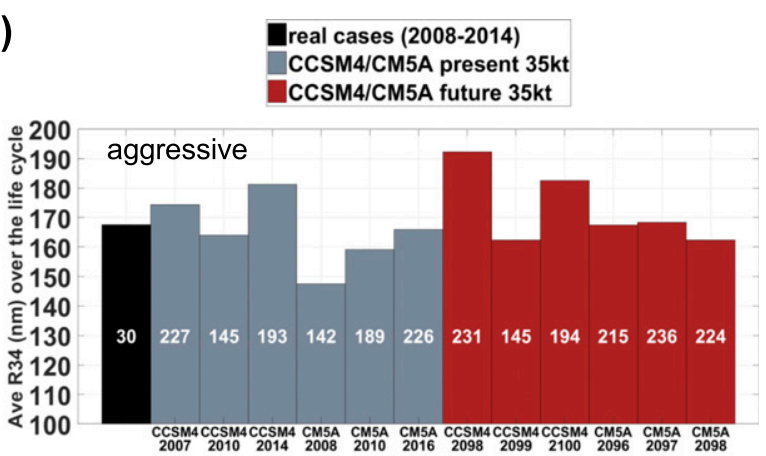

d)

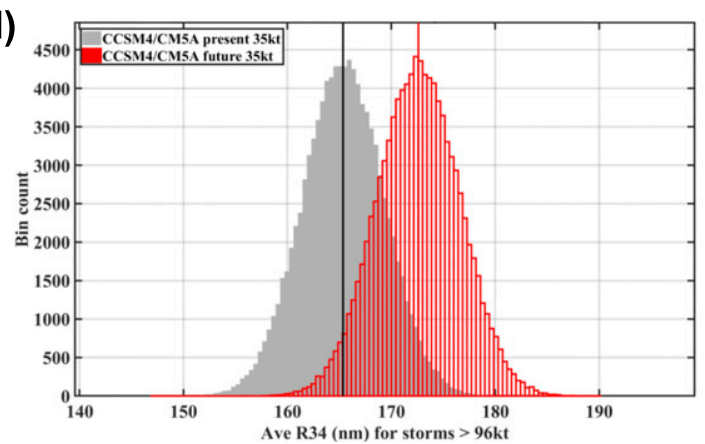

FIG. 17. (a) For the conservative configuration, the average R34 ( $\mathrm{nmi}$ ) for those TCs whose MWS is $>96 \mathrm{kt}$ at some point during the life cycle. Otherwise, as in Fig. 12a. (b) As in (a), but for the aggressive configuration. The black bar represents the simulations for the realworld cases. (c) Significance test results corresponding to (a). Otherwise as in Fig. 12c. See text for further details. (d) Significance test results corresponding to (b).

conservative when viewed through the lens of WestPac SSTs. Hence, the seeding experiments are repeated for four supplemental years to allow further assessment of the possible sensitivity of the results to year selection and interannual variability. These supplemental seeding experiments are carried out using the same methodology as the others, but are run only for the conservative configuration. A survey of the results for the supplemental experiments is presented in Fig. 19, emphasizing three metrics including the life cycle maximum intensity and average $\tau$ of storms that exceed $96 \mathrm{kt}$ and the average first landfall intensity of storms that exceed 64-kt intensity at landfall. For all three of these metrics, the supplemental results reaffirm the earlier results. Namely, the future simulations yield considerably more storms that exceed 96-kt intensity, and these storms exhibit notably greater average life cycle maximum intensity and tend to spend more time above the 96-kt intensity threshold (Figs. 19a,b). Also, the future simulations yield more storms that make landfall at $>64-\mathrm{kt}$ intensity, and the average landfall intensity of these storms is appreciably greater (Fig. 19c).

\section{i. Intensity bias correction}

Quantile-matching results are presented in Fig. 20 for the case where the COAMPS-TC forecast intensity for the CCSM4/CM5A present years and the 2008-14 best track intensity are partitioned into 99 quantiles. For both the conservative and aggressive configurations, the bin-average points lie below the $45^{\circ}$ line of the plot for all intensities, implying that the forecast intensity tends to underestimate the observed intensity. Additionally, the points progressively diverge to the lower right of the $45^{\circ}$ line as the intensity increases, meaning that the forecast's tendency to underestimate gets more pronounced as the intensity increases. This behavior can be quantified to first approximation by least squares linear fits to the data points, shown as solid lines in Fig. 20. The equation of the fit to the conservative configuration data points is

$$
c_{\text {cons }}=1.199 \times f_{\text {cons }}+12.429,
$$

where $f_{\text {cons }}\left(c_{\text {cons }}\right)$ is the forecast (observed) intensity and $f_{\text {cons }}$ is on the interval [16,124 ] (kt), and the equation of the fit to the aggressive-configuration data points is

$$
c_{\text {aggr }}=1.081 \times f_{\text {aggr }}+5.139,
$$

where $f_{\text {aggr }}$ is on the interval $[18,133](\mathrm{kt})$. Considering the conservative configuration case, the fit gives a correction of near $37 \mathrm{kt}$ when the forecast intensity is $124 \mathrm{kt}$. If it is assumed that COAMPS-TC exhibits a similar bias 
a)

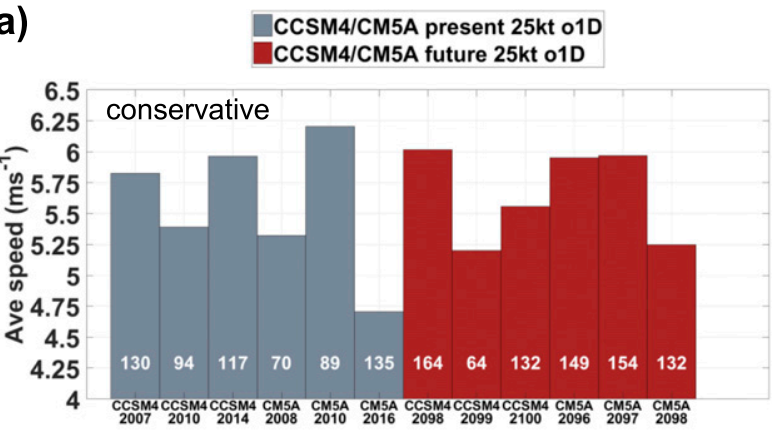

c)

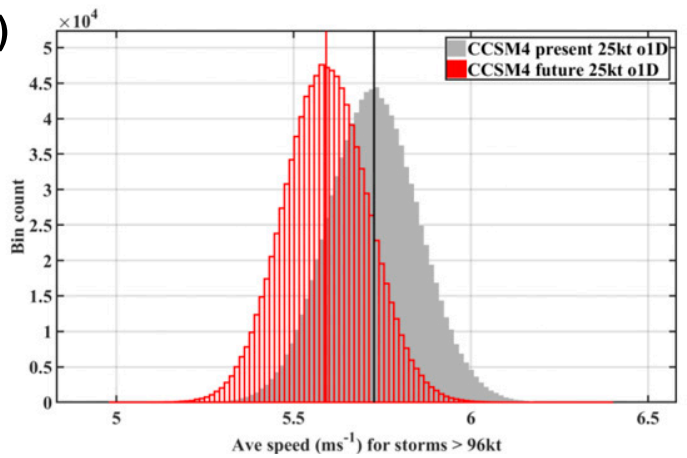

b)

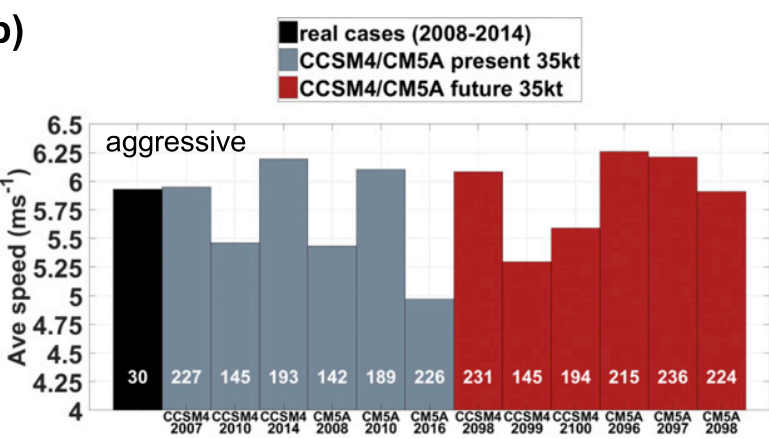

d)

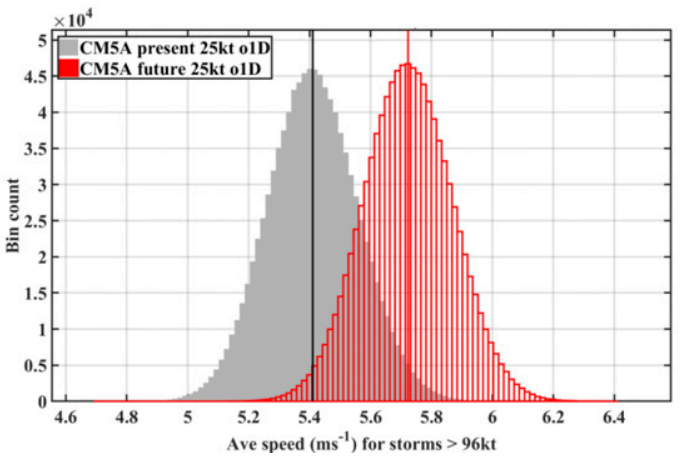

FIG. 18. (a) For the conservative configuration, the average storm speed $\left(\mathrm{m} \mathrm{s}^{-1}\right)$ for those TCs whose MWS is $>96 \mathrm{kt}$ at some point during the life cycle. Otherwise as in Fig. 12a. (b) As in (a), but for the aggressive configuration. The black bar represents the simulations for the real-world cases. (c) Significance test results corresponding to (a), but considering only the CCSM4 present years vs the CCSM4 future years. Otherwise as in Fig. 12c. See text for further details. (d) Significance test results corresponding to (a), but considering only the CM5A present years vs the CM5A future years.

toward underestimation of intensity in the large-scale environments of the future extreme climates, an assumption that does not seem unreasonable, then the possible maximum storm intensities in the future climates may be substantially greater than those seen in these COAMPS-TC simulations. In particular, if one takes the $37 \mathrm{kt}$ correction associated with the highest value of $f_{\text {cons }}$ in Eq. (1) (i.e., $124 \mathrm{kt}$ ) and applies this $37 \mathrm{kt}$ correction to the $155-\mathrm{kt}$ MWS values seen in the uncorrected COAMPS-TC simulations for the futureclimate years, then the implication is that the maximum storm intensity could approach $\sim 190-195 \mathrm{kt}$.

\section{Conclusions}

A variant of downscaling is devised to explore the properties of TCs originating in the open ocean of the WestPac region under extreme climates. This variant applies a seeding strategy in large-scale environments simulated by CMIP5 climate-model integrations together with an embedded, high-resolution, operational, convection-permitting NWP model. Two particular climate integrations are employed, one taken from a model with a low climate sensitivity (the NCAR
CCSM4) and the other from a model with a high climate sensitivity (the IPSL CM5A). Both integrations are under the RCP8.5 forcing scenario. Collectively, eight years are sampled from the present-day portion of the climate integrations and eight years are sampled from the late twenty-first century portion of the integrations. Each 8-yr test period is split into a 6-yr set that forms the basis of the main analysis and a 2-yr set that forms the basis of a supplemental sensitivity analysis. For each year, seed vortices are applied every five days over the months August-October for a total of 285 seeds per year and 2280 seeds per each 8 -yr test period. The seeds are nonrandomly placed in a small, fixed source region in the WestPac. Each seed is evolved for the duration of its life cycle or for $360 \mathrm{~h}$, whichever is shorter, with the COAMPS-TC operational TC NWP model in a nestedgrid configuration with a $5-\mathrm{km}$ resolution on the innermost grid. The seeded simulations are carried out in their entirety for two different simulation configurations: a conservative configuration and an aggressive configuration. These different configurations facilitate an examination of the sensitivity of the simulations to seed strength and ocean mixing. As the main point of comparison, the seeded simulations for the future-climate 
a)

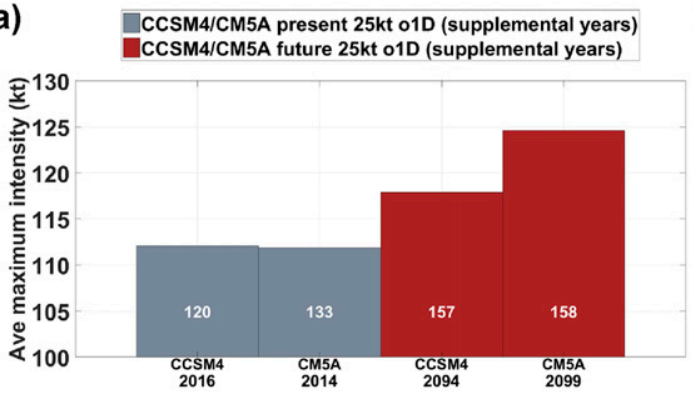

c)
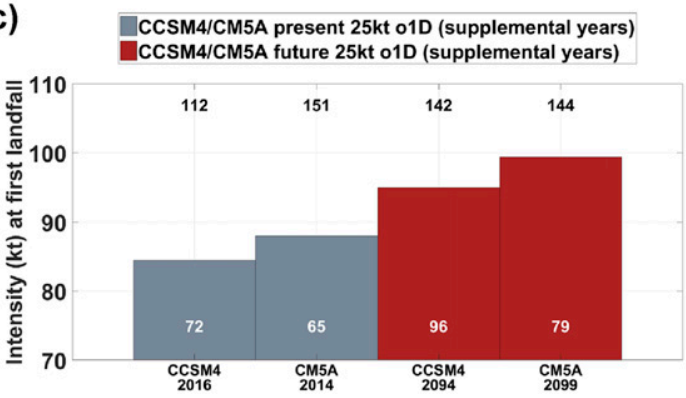

b)

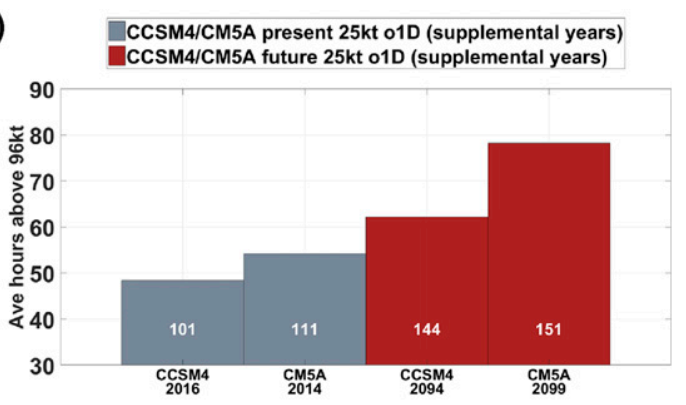

FIG. 19. For the supplemental years and the conservative configuration. (a) The average life cycle maximum intensity for those TCs whose MWS is $>96 \mathrm{kt}$ at some point during the life cycle. Otherwise as in Fig. 12a. (b) The average number of hours per life cycle that a TC simulation maintains an MWS $>96 \mathrm{kt}$, considering only those TCs whose MWS is $>96 \mathrm{kt}$ at some point during the life cycle. Otherwise as in Fig. 15a. (c) The average landfall intensity (denoted by the color bars), considering only those TCs whose MWS is $>64 \mathrm{kt}$ at landfall. Maximum landfall intensity is given by the numbers in black across the top of the plot. Otherwise as in Fig. 16a.

test period are contrasted with those for the presentclimate test period. The seeded simulations are also compared against a set of unseeded simulations for real-world TC cases that are observed over the period 2008-14 and that are of similar WestPac origin. Because the seeding process introduces nonnative vortices into the large-scale environments, the simulation results are not used to make inferences about the frequency of TCs in the futureclimate environments. Inferences are restricted to the topics of intensity and track over the storm life cycle.

Differences between the TCs in the future and present simulations are most apparent in three metrics: Life cycle maximum intensity, average hours per life cycle that intensity exceeds $96 \mathrm{kt}$, and average intensity at first landfall. In particular, the future simulations yield considerably more TCs that exceed 96-kt intensity, and these TCs exhibit notably greater average life cycle maximum intensity and tend to spend more time above the 96-kt intensity threshold. Also, the future simulations yield more TCs that make landfall at $>64-\mathrm{kt}$ intensity, and the average landfall intensity of these storms is appreciably greater. These findings are reinforced by statistical analysis based on a stringent implementation of the bootstrap as well as by the supplemental sensitivity analysis. Further, the results hold for both the conservative and aggressive simulation configurations, and generally are consistent with other recent downscaling literature.
Importantly, the stronger storms in the future are not confined to the open ocean. Rather, intense landfalling storms are seen in most of the seeded-simulation years. These more intense landfalling storms are aided by extensive warming of the WestPac SST (which tends to increase potential intensity) and reduced vertical wind shear in the vicinity of Japan and the Korean Peninsula. Some interannual variability is apparent in the intensity and landfall results, related to notable interannual changes in the genesis index and potential intensity. As might be expected, those years with the more extensive warming of SST are more conducive to intense storms.

In concert with the above intensity and landfall results, the seeded simulations suggest that the maximum storm intensity for a given latitude will be greater in the future climates than in present day. Equivalently, they suggest that storms of a given intensity will be found notably farther north in the future climates. These results echo those of Roberts et al. (2015), Kossin et al. (2016), and others. Considering storm size in terms of the radius of 34-kt wind speed, the simulations suggest that the average size of major storms of category 3 or greater may tend to be slightly larger in the future climates. The above suggestions are seen in simulations from both the conservative and aggressive configurations and in simulations from both the CCSM4 and CM5A data. 


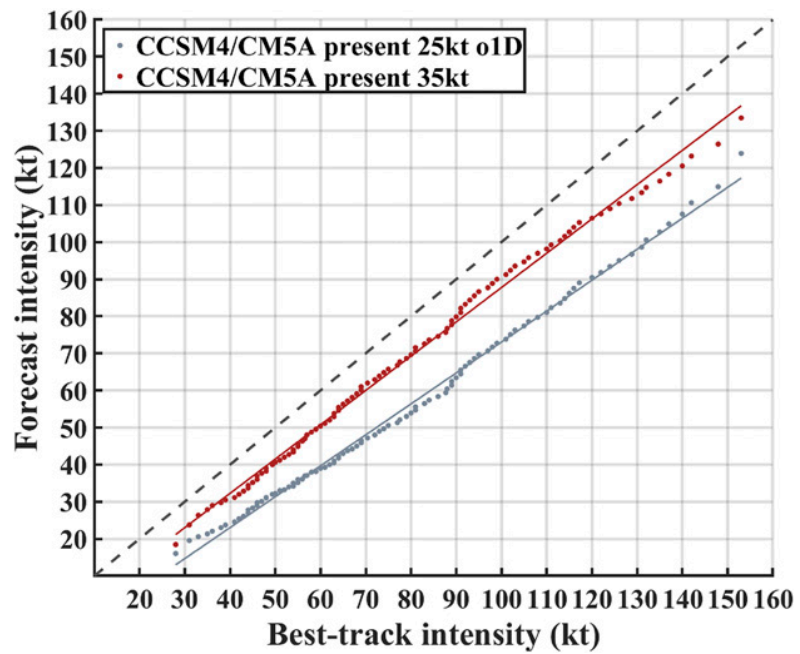

FIG. 20. Quantile comparison of the COAMPS-TC forecast MWS for the six present years of climate-model data (ordinate) and the observed (best track) MWS for real-world WestPac TCs in the period 2008-14 (abscissa). Gray (red) coloring indicates results for the conservative (aggressive) configuration. Filled circles indicate the bin-average MWS within the bins defined by respective quantiles. Solid lines are the ordinary least squares best-fit line to the bin-average MWS data points. Dashed line is for reference.

There are two metrics for which the results do show notable model dependence: The average latitude of the life cycle maximum intensity and the average storm speed. Regarding the former, the simulations based on CCSM4 data show no significant difference between the present and future TCs, whereas the simulations based on CM5A data show a clear northward displacement for future TCs. Considering the storm speed, the simulations based on CCSM4 data suggest a slight (and not statistically significant) decrease in storm speed $\left(\approx-0.1 \mathrm{~m} \mathrm{~s}^{-1}\right.$ for the conservative configuration) for those TCs that exceed 96-kt intensity, whereas the simulations based on CM5A data indicate a modest but statistically significant increase in storm speed $\left(\approx+0.3 \mathrm{~m} \mathrm{~s}^{-1}\right.$ for the conservative configuration) for such storms. Thus, there is insufficient evidence to claim that either of these metrics will be different for TCs in the future climate.

The question of storm speed assumes some additional importance for the reason that slower-moving storms over seas with shallower mixed layers may exhibit a more pronounced ocean coupling effect and hence reduced intensification. To underscore this point, some recent literature suggests there is a good chance that TCs will in fact move more slowly in future climates (Kossin 2018). Likewise, as noted earlier in the discussion of the parameterized ocean coupling in section $2 \mathrm{a}(4)$, recent literature also suggests there is some possibility that a future climate will exhibit enhanced ocean thermal stratification (e.g., Capotondi et al. 2012; Sallee et al. 2013;
Chen and Wang 2015; Emanuel 2015; Huang et al. 2015; Mei et al. 2015; Tuleya et al. 2016). These findings suggest that it might be useful future work to apply the simulation framework of this study to a broader set of climate-model data to better resolve how storm speed combines with ocean thermal structure to influence intensification in the future climates.

Based on the application of a quantile-matching approach to intensity bias correction that compares the distribution of seeded forecasts for the present day against the distribution of best track observations of real-world storms, the seeded simulations have a pronounced tendency to underestimate the intensity of storms. As remarked in section $2 b(5)$, the version of COAMPS-TC used for this study is known to have such an underestimation bias, particularly for stronger storms. However, the nature of the large-scale environmental conditions from the CCSM4 and CM5A climate integrations may also contribute to the underestimation. Whatever the source of the underestimation, the biascorrection results imply that the potential maximum storm intensities in the future extreme climate may be substantially greater than the $155-160 \mathrm{kt}(875-880 \mathrm{hPa})$ maximum intensities seen in these COAMPS-TC seeded simulations. In the case of maximum wind speed, the potential intensities could be as much as $37 \mathrm{kt}$ greater than in the seeded simulations using the conservative configuration, or more than $190 \mathrm{kt}$. This result is in agreement with the $97 \mathrm{~m} \mathrm{~s}^{-1}$ maximum intensities suggested by the simulations of Korty et al. (2017).

The above conclusions are tempered somewhat by the fact that the ocean thermal stratification is not explicitly modeled in this study. As noted above in the discussion of storm speed and earlier in the discussion of parameterized ocean coupling in section $2 \mathrm{a}(4)$, recent literature suggests there is some possibility that a future climate will exhibit enhanced ocean thermal stratification. Such an enhanced stratification may facilitate a stronger ocean coupling effect, which would offset some of the intensification seen in the future climates. In particular, estimates suggest a stronger ocean coupling effect probably would cause a $10 \%-20 \%$ reduction in the intensification related to climate change (e.g., Emanuel 2015; Tuleya et al. 2016). However, there is considerable variability in the future ocean thermal stratification of the WestPac region across the CMIP5 models (e.g., Huang et al. 2015) and the CMIP5 models also may be biased too shallow in terms of mixed layer depth (e.g., Sallee et al. 2013; Huang et al. 2014). As a result, it remains an open question as to whether a stronger ocean coupling effect will actually materialize in a future climate and, if it does, precisely how strong it will be. The uncertainty surrounding the ocean coupling effect, together with the effect's potential importance, suggests 
that it would be useful in future work to incorporate a model of ocean thermal stratification in the simulation framework of this study. Other possible future work includes revisiting various cases using a multiphysics ensemble to assess the importance of physics uncertainty. Also, the seeding experiments should be carried out using a seeding region that encompasses the South China Sea, as this area is an important source of landfalling TCs.

Acknowledgments. The comments of four anonymous reviewers were instrumental in improving this study. The study was supported by the Chief of Naval Research (Program Element 0601153N). EAH acknowledges support from the Office of Naval Research (ONR) Grant N0001417WX01042. The DOD High Performance Computing program at NAVO DSRC provided the computing resources.

\section{REFERENCES}

Alessandrini, S., L. D. Monache, C. M. Rozoff, and W. E. Lewis, 2018: Probabilistic prediction of tropical cyclone intensity with an analog ensemble. Mon. Wea. Rev., 146, 1723-1744, https:// doi.org/10.1175/MWR-D-17-0314.1.

Bender, M. A., T. R. Knutson, R. E. Tuleya, J. J. Sirutis, G. A. Vecchi, S. T. Garner, and I. M. Held, 2010: Model impact of anthropogenic warming on the frequency of intense Atlantic hurricanes. Science, 327, 454-458, https://doi.org/10.1126/ science. 1180568 .

Bengtsson, L., K. I. Hodges, M. Esch, N. Keenlyside, L. Kornblueh, J.-J. Luo, and T. Yamagata, 2007: How may tropical cyclones change in a warmer climate? Tellus, 59A, 538-560, https:// doi.org/10.1111/j.1600-0870.2007.00251.x.

Bentley, J., 1999: Programming Pearls. 2nd ed. Addison-Wesley, 256 pp.

Bister, M., and K. A. Emanuel, 2002: Low frequency variability of tropical cyclone potential intensity 1 . Interannual to interdecadal variability. J. Geophys. Res., 107, 4801, https://doi.org/ 10.1029/2001JD000776.

Camargo, S. J., 2013: Global and regional aspects of tropical cyclone activity in the CMIP5 models. J. Climate, 26, 98809902, https://doi.org/10.1175/JCLI-D-12-00549.1.

_ K. A. Emanuel, and A. H. Sobel, 2007: Use of a genesis potential index to diagnose ENSO effects on tropical cyclone genesis. J. Climate, 20, 4819-4834, https://doi.org/10.1175/ JCLI4282.1.

Capotondi, A., M. A. Alexander, N. A. Bond, E. N. Curchitser, and J. D. Scott, 2012: Enhanced upper ocean stratification with climate change in the CMIP3 models. J. Geophys. Res., 117, C04031, https://doi.org/10.1029/2011JC007409.

Chen, C., and G. Wang, 2015: Role of North Pacific mixed layer in the response of SST annual cycle to global warming. J. Climate, $\mathbf{2 8}$, 9451-9458, https://doi.org/10.1175/JCLI-D-14-00349.1.

Choi, K.-S., and Y.-M. Cha, 2012: Change in future tropical cyclone activity over the western North Pacific under global warming scenario. Nat. Hazards, 64, 1125-1140, https://doi.org/10.1007/ s11069-012-0285-3.

Donelan, M. A., B. K. Haus, N. Reul, W. J. Plant, M. Stiassnie, and H. C. Graber, 2004: On the limiting aerodynamic roughness of the ocean in very strong winds. Geophys. Res. Lett., 31, L18306, https://doi.org/10.1029/2004GL019460.
Doyle, J. D., and Coauthors, 2014: Tropical cyclone prediction using COAMPS-TC. Oceanography, 27 (3), 104-115, https:// doi.org/10.5670/oceanog.2014.72.

Dufresne, J.-L., and Coauthors, 2013: Climate change projections using the IPSL-CM5 Earth System Model: From CMIP3 to CMIP5. Climate Dyn., 40, 2123-2165, https://doi.org/10.1007/ s00382-012-1636-1.

Emanuel, K., 1995: The behavior of a simple hurricane model using a convective scheme based on subcloud-layer entropy equilibrium. J. Atmos. Sci., 52, 3960-3968, https://doi.org/10.1175/ 1520-0469(1995)052<3960:TBOASH>2.0.CO;2.

, 2006: Climate and tropical cyclone activity: A new model downscaling approach. J. Climate, 19, 4797-4802, https:// doi.org/10.1175/JCLI3908.1.

, 2013: Downscaling CMIP5 climate models shows increased tropical cyclone activity over the 21st century. Proc. Natl. Acad. Sci. USA, 110, 12 219-12 224, https://doi.org/10.1073/pnas.1301293110.

2015: Effect of upper-ocean evolution on projected trends in tropical cyclone activity. J. Climate, 28, 8165-8170, https:// doi.org/10.1175/JCLI-D-15-0401.1.

- , and D. S. Nolan, 2004: Tropical cyclone activity and the global climate system. 26th Conf. on Hurricanes and Tropical Meteorology, Miami, FL, Amer. Meteor. Soc., 10A.2, https:// ams.confex.com/ams/pdfpapers/75463.pdf.

C. DesAutels, C. Holloway, and R. Korty, 2004: Environmental control of tropical cyclone intensity. J. Atmos. Sci., 61, 843-858, https://doi.org/10.1175/1520-0469(2004)061<0843: ECOTCI $>2.0 . \mathrm{CO} ; 2$.

—, R. Sundararajan, and J. Williams, 2008: Hurricanes and global warming: Results from downscaling IPCC AR4 simulations. Bull. Amer. Meteor. Soc., 89, 347-367, https://doi.org/ 10.1175/BAMS-89-3-347.

Fujiwhara, S., 1921: The natural tendency towards symmetry of motion and its application as a principle in meteorology. Quart. J. Roy. Meteor. Soc., 47, 287-293, https://doi.org/ 10.1002/qj.49704720010.

Gall, R., J. Franklin, F. Marks, E. N. Rappaport, and F. Toepfer, 2013: The Hurricane Forecast Improvement Project. Bull. Amer. Meteor. Soc., 94, 329-343, https://doi.org/10.1175/ BAMS-D-12-00071.1.

Gent, P. R., and Coauthors, 2011: The Community Climate System Model Version 4. J. Climate, 24, 4973-4991, https://doi.org/ 10.1175/2011JCLI4083.1.

Hendricks, E. A., M. S. Peng, and T. Li, 2013: Evaluation of multiple dynamic initialization schemes for tropical cyclone prediction. Mon. Wea. Rev., 141, 4028-4048, https://doi.org/ 10.1175/MWR-D-12-00329.1.

Hill, K. A., and G. M. Lackmann, 2011: The impact of future climate change on TC intensity and structure: A downscaling approach. J. Climate, 24, 4644-4661, https://doi.org/10.1175/ 2011JCLI3761.1.

Hogan, T. F., and Coauthors, 2014: Oceanography, 27 (3), 116-125, https://doi.org/10.5670/oceanog.2014.73.

Huang, C. J., F. Qiao, and D. Dai, 2014: Evaluating CMIP5 simulations of mixed layer depth during summer. J. Geophys. Res. Oceans, 119, 2568-2582, https://doi.org/10.1002/ 2013JC009535.

Huang, P., I.-I. Lin, C. Chou, and R.-H. Huang, 2015: Change in ocean subsurface environment to suppress tropical cyclone intensification under global warming. Nat. Commun., 6, 7188, https://doi.org/10.1038/ncomms8188.

Jin, Y., W. T. Thompson, S. Wang, and C.-S. Liou, 2007: A numerical study of the effect of dissipative heating on tropical 
cyclone intensity. Wea. Forecasting, 22, 950-966, https:// doi.org/10.1175/WAF1028.1.

Khairoutdinov, M. F., and K. Emanuel, 2013: Rotating radiativeconvective equilibrium simulated by a cloud-resolving model. J. Adv. Model. Earth Syst., 5, 816-825, https://doi.org/10.1002/ 2013MS000253.

Knapp, K. R., M. C. Kruk, D. H. Levinson, H. J. Diamond, and C. J. Neumann, 2010: The International Best Track Archive for Climate Stewardship (IBTrACS) unifying tropical cyclone data. Bull. Amer. Meteor. Soc., 91, 363-376, https://doi.org/ 10.1175/2009BAMS2755.1.

Knutson, T. R., J. J. Sirutis, S. T. Garner, G. A. Vecchi, and I. M. Held, 2008: Simulated reduction in Atlantic hurricane frequency under twenty-first-century warming conditions. Nat. Geosci., 1, 359-364, https://doi.org/10.1038/ngeo202.

_ - and Coauthors, 2013: Dynamical downscaling projections of twenty-first-century Atlantic hurricane activity: CMIP3 and CMIP5 model-based scenarios. J. Climate, 26, 6591-6617, https://doi.org/10.1175/JCLI-D-12-00539.1.

—, J. J. Sirutis, M. Zhao, R. E. Tuleya, M. Bender, G. A. Vecchi, G. Villarini, and D. Chavas, 2015: Global projections of intense tropical cyclone activity for the late twenty-first century from dynamical downscaling of CMIP5/RCP4.5 scenarios. J. Climate, 28, 7203-7224, https://doi.org/10.1175/JCLI-D-15-0129.1.

Korty, R. L., K. A. Emanuel, M. Huber, and R. A. Zamora, 2017: Tropical cyclones downscaled from simulations with very high carbon dioxide levels. J. Climate, 30, 649-667, https://doi.org/ 10.1175/JCLI-D-16-0256.1.

Kossin, J. P., 2018: A global slowdown of tropical-cyclone translation speed. Nature, 558, 104-107, https://doi.org/10.1038/ s41586-018-0158-3.

_ K. A. Emanuel, and S. J. Camargo, 2016: Past and projected changes in western North Pacific tropical cyclone exposure. J. Climate, 29, 5725-5739, https://doi.org/10.1175/JCLI-D-16-0076.1.

Liou, C.-S., and K. D. Sashegyi, 2012: On the initialization of tropical cyclones with a three-dimensional variational analysis. Nat. Hazards, 63, 1375-1391, https://doi.org/10.1007/ s11069-011-9838-0.

McLay, J. G., C. A. Reynolds, E. Satterfield, and D. Hodyss, 2016: Changes to intrinsic weather forecast uncertainty in one scenario of extreme future climate. Quart. J. Roy. Meteor. Soc., 142, 2102-2118, https://doi.org/10.1002/qj.2806.

Mei, W., S.-P. Xie, F. Primeau, J. C. McWilliams, and C. Pasquero, 2015: Northwestern Pacific typhoon intensity controlled by changes in ocean temperatures. Sci. Adv., 1, e1500014, https:// doi.org/10.1126/sciadv.1500014.

Murakami, H., B. Wang, and A. Kitoh, 2011: Future change of western North Pacific typhoons: Projections by a $20-\mathrm{km}-\mathrm{mesh}$ global atmospheric model. J. Climate, 24, 1154-1168, https:// doi.org/10.1175/2010JCLI3723.1.

— and Coauthors, 2012: Future changes in tropical cyclone activity projected by the new high-resolution MRI-AGCM. J. Climate, 25, 3237-3260, https://doi.org/10.1175/JCLI-D-11-00415.1.

Nakamura, R., T. Shibayama, M. Esteban, and T. Iwamoto, 2016: Future typhoon and storm surges under different global warming scenarios: case study of Typhoon Haiyan (2013). Nat. Hazards, 82, 1645-1681, https://doi.org/10.1007/s11069-016-2259-3.

Oouchi, K., J. Yoshimura, H. Yoshimura, R. Mizuta, S. Kusunoki, and A. Noda, 2006: Tropical cyclone climatology in a global-warming climate as simulated in a $20 \mathrm{~km}$-mesh global atmospheric model: Frequency and wind intensity analyses. J. Meteor. Soc. Japan, 84, 259-276, https://doi.org/10.2151/jmsj.84.259.
Roberts, M. J., and Coauthors, 2015: Tropical cyclones in the UPSCALE ensemble of high-resolution global climate models. J. Climate, 28, 574-596, https://doi.org/10.1175/JCLI-D-1400131.1.

Sallee, J.-B., E. Shuckburgh, N. Bruneau, A. J. S. Meijers, T. J. Bracegirdle, and Z. Wang, 2013: Assessment of Southern Ocean mixed layer depths in CMIP5 models: Historical bias and forcing response. J. Geophys. Res. Oceans, 118, 1845-1862, https://doi.org/10.1002/ jgrc.20157.

Simpson, R. H., and H. Riehl, 1981: The Hurricane and Its Impact. LSU Press, $398 \mathrm{pp}$.

Sun, Y., and Coauthors, 2017: Impact of ocean warming on tropical cyclone size and its destructiveness. Sci. Rep., 7, 8154, https:// doi.org/10.1038/s41598-017-08533-6.

Swiss Re, 2017: Natural catastrophes and man-made disasters in 2016: A year of widespread disasters. Swiss Re Sigma 2, 43 pp. [Available online at https://www.preventionweb.net/publications/view/52534.]

Taylor, K. E., R. J. Stouffer, and G. A. Meehl, 2012: An overview of CMIP5 and the experiment design. Bull. Amer. Meteor. Soc., 93, 485-498, https://doi.org/10.1175/BAMS-D-11-00094.1.

Tsuboki, K., M. K. Yoshioka, T. Shinoda, M. Kato, S. Kanada, and A. Kitoh, 2015: Future increase of supertyphoon intensity associated with climate change. Geophys. Res. Lett., 42, 646652, https://doi.org/10.1002/2014GL061793.

Tuleya, R. E., M. Bender, T. R. Knutson, J. J. Sirutis, B. Thomas, and I. Ginis, 2016: Impact of upper tropospheric temperature anomalies and vertical wind shear on tropical cyclone evolution using an idealized version of the operational GFDL hurricane model. J. Atmos. Sci., 73, 3803-3820, https://doi.org/ 10.1175/JAS-D-16-0045.1.

Vial, J., J.-L. Dufresne, and S. Bony, 2013: On the interpretation of intermodel spread in CMIP5 climate sensitivity estimates. Climate Dyn., 41, 3339-3362, https://doi.org/10.1007/s00382-013-1725-9.

Villarini, G., and G. A. Vecchi, 2013: Projected increases in North Atlantic tropical cyclone intensity from CMIP5 models. J. Climate, 26, 3231-3240, https://doi.org/10.1175/JCLI-D-12-00441.1.

Walsh, K. E., and Coauthors, 2015: Tropical cyclones and climate change. Wiley Interdiscip. Rev.: Climate Change, 7, 65-89, https://doi.org/10.1002/wcc.371.

Wang, C., and L. Wu, 2015: Influence of future tropical cyclone track changes on their basin-wide intensity over the western North Pacific: Downscaled CMIP5 projections. Adv. Atmos. Sci., 32, 613-623, https://doi.org/10.1007/s00376-014-4105-4.

Wang, H.-J., R.-H. Zhang, J. Cole, and F. Chavez, 1999: El Niño and the related phenomenon Southern Oscillation (ENSO): The largest signal in interannual climate variation. Proc. Natl. Acad. Sci. USA, 96, 11 071-11 072, https://doi.org/10.1073/pnas.96.20.11071.

Williams, D. N., B. N. Lawrence, M. Lautenschlager, D. Middleton, and V. Balaji, 2011: The Earth System Grid Federation: Delivering globally accessible petascale data for CMIP5. Proc. Asia-Pac. Adv. Network, 32, 121-130, https:// doi.org/10.7125/APAN.32.15.

Yokoi, S., Y. N. Takayabu, and H. Murakami, 2013: Attribution of projected future changes in tropical cyclone passage frequency over the western North Pacific. J. Climate, 26, 4096-4111, https://doi.org/10.1175/JCLI-D-12-00218.1.

Yoshimura, J., and M. Sugi, 2005: Tropical cyclone climatology in a high-resolution AGCM-Impacts of SST warming and $\mathrm{CO}_{2}$ increase. SOLA, 1, 133-136, https://doi.org/10.2151/sola.2005-035.

Zhao, M., and I. M. Held, 2010: An analysis of the effect of global warming on the intensity of Atlantic hurricanes using a GCM with statistical refinement. J. Climate, 23, 6382-6393, https:// doi.org/10.1175/2010JCLI3837.1. 